\title{
FONÉTICA ARTICULATORIA, RUIDO E INTELIGIBILIDAD EN ESPAÑOL*
}

\section{ARTICULATORY PHONETICS, NOISE AND INTELIGIBILITY IN SPANISH}

\author{
JORGE SOMMERHOFF HYDE \\ Universidad Austral de Chile \\ jsommerh@uach.cl
}

\section{CLAUDIA ROSAS AGUILAR}

Universidad Austral de Chile

claudiarosas@uach.cl

\section{RESUMEN}

El propósito de este estudio es evaluar el efecto de los rasgos articulatorios en la percepción de los enunciados en español en condiciones de ruido, es decir, con una distorsión generada en el dominio de la frecuencia. Para ello, se utilizó un corpus fonéticamente balanceado de 920 logatomos de estructura consonante-vocal-consonante (CVC) que un hablante masculino emitió, bajo condiciones de ruido blanco, a un grupo de 17 auditores en cuatro sesiones separadas por un periodo de una semana cada una. Las respuestas fueron tabuladas en una plantilla Excel ad hoc y clasificadas de acuerdo al tipo de respuesta en: omitidas, confundidas y acertadas. Posteriormente, se comparó la palabra confundida u omitida con la palabra correcta. Los resultados demuestran que el ruido afecta de manera desigual a los componentes de la estructura del logatomo CVC, al tipo de sonido consonántico o vocálico y al tipo de sonido con el que se confunde cada vocal y cada consonante en particular.

Palabras clave: Fonética perceptual, inteligibilidad, ruido, claves articulatorias.

\section{ABSTRACT}

The purpose of this study is to evaluate the effect of articulatory features on the perception the utterances in Spanish under noise conditions, that is, with a distortion generated in the frequency domain. For this purpose, a phonetically balanced corpus of 920 logatomos

* Fondecyt Regular 1090249. "Elaboración de un instrumento para medir la inteligibilidad del habla en español mediante el uso de logatomos". Inv. Responsable: Jorge Sommerhoff/Coi: Claudia Rosas. 
consonant-vocal-consonant (CVC) structure was used that a male speaker emitted, under conditions of white noise, to a group of 17 auditors in four sessions separated by a period of one week each. The answers were tabulated in an ad hoc Excel template and classified according to the response type in: omitted, confused and correct. Subsequently, the confused or omitted word was compared with the correct word. The results show that noise unequally affects the components of the CVC logatom structure, the consonant or vowel type of sound, and the type of sound with which each vowel and each particular consonant is confused.

Keywords: Perceptual phonetics, intelligibility, noise, articulatory keys.

Recibido: 01.04.2017. Aceptado: 11.08.2017.

\section{INTRODUCCIÓN}

Tos estudios sobre la inteligibilidad del habla se han centrado tradicionalmente en la medición de la calidad acústica de los recintos (Marxer, Barker, Martin y García, 2016) y, más recientemente, en los diversos tipos de condicionamientos que influyen en la detección de la señal lingüística o no lingüística. Con respecto a los rasgos lingüísticos, para el inglés, Parikh y Loizou (2005) evaluaron el efecto acústico y perceptual del ruido multihablante y en forma de habla (de -5 a + $10 \mathrm{~dB} S / \mathrm{N})$ de las vocales en el interior de palabras, en un corpus fonéticamente balanceado; y de las consonantes oclusivas, en pseudopalabras de estructura vocalconsonante-vocal VCV. Los análisis acústicos indicaron que el F1 fue hallado más confiable que el F2 y las mayores diferencias de envolvente espectral entre los espectros ruidosos y limpios de vocales se produjeron en la banda de frecuencia media; en tanto que el reconocimiento de la consonante oclusiva se mantuvo alto incluso a $-5 \mathrm{~dB}$ a pesar de la interrupción de las señales de oclusión debido al ruido aditivo, lo que sugiere, en opinión de los propios autores, que los oyentes deben estar confiando en otras claves para identificar oclusivas, como las transiciones de formantes. En otro estudio, Ziegler, Pech, George y Lorenzi (2009), con niños disléxicos a los que se presentaron pseudopalabras con el mismo tipo de estructura $\mathrm{VCV}$, con las 16 consonantes del inglés enmascaradas con ruido estacionario y fluctuante en forma de habla, encontraron que el lugar de articulación fue más afectado que la sonoridad o el modo de articulación. Cunningham, Nicol, Zecker, Bradlow y Kraus (2001) estudiaron una gama de sílabas sintetizadas desde /ada/ a /aga/ con ruido blanco en niños con patologías de aprendizaje, cuya dificultad para ser reconocidas en este tipo de auditores ya había sido probada, y demostraron que el incremento de la duración de espacio blanco en la fase de cierre y el incremento de la intensidad de la barra de explosión en la fase de apertura mejoraban la percepción de los sonidos del habla, al igual que las representaciones 
neurofisiológicas de las características degradadas por el ruido blanco. Además, encontraron que el incremento de intensidad de la barra de explosión reducía el enmascaramiento producido en la vocal siguiente. Tessa y Atagi (2015) investigaron cómo la condición no nativo y la condición de ruido afectan el reconocimiento de palabras de niños y adultos, tanto por separado como en combinación. Los estímulos incluyeron 80 oraciones de la prueba de audición en ruido para niños (HINT-C) (Nilsson, Soli y Sullivan, 1994), presentados en 4 bloques de 20 oraciones cada uno: nativo en silencio, nativo en ruido, no nativo en silencio y no nativo en ruido. Los resultados demostraron que el reconocimiento de palabras era muy preciso con sólo una condición adversa de audición (no nativo o ruido), pero mostró decrementos sustanciales cuando los desafíos de audición se combinaron. Específicamente, los niños tenían más dificultad que los adultos que identificaban el habla nativa en el ruido o el habla no nativa en el silencio, pero que se desempeñaban particularmente mal con el habla no nativa en el ruido. Los autores sugieren como explicación que las habilidades de reconocimiento de las palabras de los niños, tanto en la adversidad ambiental como en la del hablante, aún se están desarrollando en los primeros años de edad escolar. En otro estudio de propósito parcialmente similar, Liu y Jin (2015) investigaron si los oyentes nativos chinos y coreanos procesaban el habla de manera diferente a la de los oyentes no nativos en la detección de vocales y de sonidos no vocálicos (tonos puros y complejos) en condición de ruido. Descubrieron que los oyentes chinos y coreanos actuaron de manera semejante al detectar sonidos no vocálicos en ruido, mientras que al identificar sonidos de vocales coreanas en ruido, los oyentes coreanos superaron a sus homólogos chinos. Los autores atribuyen esto a que las señales estaban en el discurso nativo de los oyentes coreanos, cuyo sistema vocálico más denso, y por ende, su mayor sensibilidad ante las vocales, facilitaría su detección, incluso, en condiciones adversas.

Léger, Reed, Desloge, Swaminathan y Braida (2015), desde una perspectiva más física, se interesaron por examinar la capacidad para identificar consonantes en estructura VCV de oyentes normales y con impedimentos auditivos en ruido estacionario y ruido de baja frecuencia con interrupciones. Más allá de que los hablantes sin problemas auditivos obtuvieran un mejor reconocimiento en ruido, lo interesante y sugestivo del experimento es que los hablantes con problemas auditivos obtuvieran un mejor comportamiento relativo en ruido estacionario que en ruido multihablante, hecho que los autores atribuyen a los efectos de nivel asociados con la manera en la que el procesamiento de estructura temporal fina (del inglés, acoustic temporal fine TFS) interactúa con la señal de ruido interrumpida, en lugar de las contribuciones de las señales de estructura temporal fina en sí.

Para el español, Marrero, Rodríguez, Igualada (2015) refieren un estudio, donde utilizando palabras en condiciones que imitaban los efectos de una hipoacusia neurosensorial sobre la señal (filtro en frecuencias altas y reverberación) los luga- 
res de articulación resultaron menos resistentes a la distorsión que los modos de articulación y las vocales obtuvieron mejor reconocimiento que las consonantes, incluidas las oclusivas.

Otra perspectiva de estudio en desarrollo se refiere a las percepciones erróneas que resultan sistemáticas en ambientes ruidosos. Para el español, encontramos un par de estudios: el de Tóth, García, Tang y Cooke (2015) que a partir de una lista de palabras frecuentes más ruido estacionario y de tipo flexible (que difería en la profundidad de la modulación temporal) en un rango de $-8 \mathrm{a}+1 \mathrm{~dB}$ con 172 estudiantes universitarios obtiene un corpus de 3.235 percepciones erróneas que se repiten entre los auditores; y el de Marxer et al. (2016) que replica el estudio anterior para el inglés, obteniendo un corpus de 3.207 percepciones erróneas consistentes. Ambos corpus están disponibles gratuitamente en línea.

\section{MÉTODOS}

\subsection{Material de habla}

El listado de palabras dictadas consistió en 920 logatomos de estructura silábica CVC consonante-vocal-consonante, agrupados en 10 listas fonéticamente balanceadas de 92 palabras cada una, basado en el corpus anterior que fue desarrollado para satisfacer la necesidad de evaluar subjetivamente la inteligibilidad del habla de español hispanoamericano (Sommerhoff y Rosas, 2012). Cada logatomo CVC incluye todas los fonemas del español hispanoamericano, exceptuando en posición inicial la consonante vibrante simple " $\mathrm{r}$ " que nunca aparece en esa posición y las consonantes alveolar aproximante sorda " $s$ ", palatal africada sorda "ch", palatal aproximante sonora "ll" y alveolar vibrante simple " $\mathrm{r}$ " en posición final, porque siempre arrojaban un alto o bajo porcentaje de aciertos -independientemente de las condiciones acústicas- o, simplemente, no aparecen en esa posición. (Ver listado en Anexo 1).

\subsection{Auditores}

Los auditores fueron estudiantes de la carrera de Pedagogía en Lenguaje y Comunicación de la Universidad Austral de Chile, oriundos de Chile y nativos de español hispanoamericano, 4 hombres y 13 mujeres, de entre 19 y 20 años, sin problemas auditivos. Todos los estudiantes fueron informados de los alcances de la investigación y consintieron en colaborar sin pago. 


\subsection{Condiciones acústicas de la sala}

Los 31.271 logatomos se dictaron en diferentes rangos de STI entre 0,4 y 0,8 : 12.874 logatomos $(41,17 \%)$ entre $04-0,5$ STI; 5.059 logatomos $(16,18 \%)$ entre 0,51-0,6 STI; 8.004 logatomos (25,60\%) entre 0,61-07 STI; 5.334 logatomos $(17,06 \%)$ entre $0,71-0,8$ STI.

\subsection{Aplicación del Test}

El test completo se aplicó en 4 sesiones separadas por un periodo de una semana cada una, durante el mes de octubre de 2015. En las sesiones 1 y 2 se administraron las listas 1 a 5 y 6 a 10, respectivamente, en sala normal; y en las sesiones 3 y 4 se administraron las listas 1 a 5 y 6 a 10, respectivamente, en sala ruido. La duración aproximada de cada sesión fue de 1 hora.

Los auditores fueron entrenados para evitar que su desempeño se viese alterado por cuestiones no auditivas. Así recibieron instrucciones sobre el tipo de estructura a escuchar, qué hacer en caso de no oír, qué grafías usar cuando los sonidos tuvieren más de una representación, básicamente. Abajo se copian las instrucciones usadas en el entrenamiento:

\section{Instrucciones}

A continuación se dictarán palabras breves, casi todas sin sentido como TAR, PUD, LAM, etc.

Escriba lo que entiende aunque no sea toda la palabra.

Si no comprende ningún sonido de la palabra, deje el casillero en blanco y continúe con la siguiente.

Para escribir las palabras, utilice las grafías del español.

En aquellos casos donde sea posible representar un sonido por más de una grafía, puede elegir cualquiera de las opciones que usted prefiera, p.e: la palabra SIL cuyo sonido inicial se puede representar con "s", "c" o "z"; el sonido final de la palabra TIR, donde se pueden usar las grafías " $r$ " o "rr"; el sonido inicial de la palabra yel, donde se puede usar: "y", "ll" o incluso "h + vocal"; el sonido inicial que aparece en KIL, donde cabe usar " $k$ ", "q + vocal" y "c"; el sonido inicial de la palabra jer, donde se pueden usar: "j”, "x", "g"; el sonido inicial 
de la palabra BID, donde se pueden usar: "b", "v" o incluso "w"; el sonido inicial de la palabra GER, donde se pueden usar: "g", "g + vocal" o incluso "w".

\subsection{Corrección de listas}

Las listas fueron corregidas de acuerdo a los criterios ortográficos mencionados arriba en dos etapas; primero, manualmente por los propios estudiantes quienes se intercambiaron las pruebas de articulación y, posteriormente, revisadas una a una por los investigadores. Las respuestas fueron vaciadas en una planilla preparada para el efecto por una asistente. El trabajo de revisión se extendió por un mes.

\section{RESULTADOS}

\subsection{A nivel general y de estructura}

A nivel completo de logatomo, de los 31.273 logatomos de estructura CVC, se constata un $2,31 \%$ de logatomos omitidos, un $22,87 \%$ de logatomos incorrectos y un $74,82 \%$ de logatomos correctos. Internamente, solo las consonantes iniciales presentaron omisiones, con un $2,29 \%$. A su vez, las vocales presentaron un $99,66 \%$ de aciertos, las consonantes iniciales, un 93,29\% y las consonantes finales, un 86,48\%. (Ver el detalle en Anexo 2).

Tabla I. Porcentajes de omisiones, errores y aciertos de los logatomos dictados.

\begin{tabular}{|l|c|}
\hline Total de logatomos dictados $=31.273$ & $100 \%$ \\
\hline Omitidos & $2,31 \%$ \\
\hline Incorrectos & $22,87 \%$ \\
\hline Correctos & $74,82 \%$ \\
\hline Consonantes iniciales omitidas & $2,29 \%$ \\
\hline Vocales omitidas & $0,00 \%$ \\
\hline Consonantes finales omitidas & $0,00 \%$ \\
\hline Consonantes iniciales confundidas & $6,72 \%$ \\
\hline Vocales confundidas & $0,35 \%$ \\
\hline Consonantes finales confundidas & $13,52 \%$ \\
\hline Consonantes iniciales acertadas & $93,29 \%$ \\
\hline Vocales acertadas & $99,66 \%$ \\
\hline Consonantes finales acertadas & $86,48 \%$ \\
\hline
\end{tabular}




\subsection{A nivel de sonido}

\subsubsection{La consonante inicial}

Las omisiones se concentraron mayoritariamente en las oclusivas sordas: la bilabial "p" con 116 casos (0,37\%), seguida de la velar "k", con 103 casos $(0,33 \%)$ y de la dental "t", con 80 casos $(0,26 \%)$. Por su parte, las tres consonantes menos omitidas fueron la palatal nasal sonora "n", con 10 casos $(0,03 \%)$, la alveolar aproximante sorda " $\mathrm{s}$ ", con 18 casos $(0,06 \%)$ y la bilabial nasal sonora "m", con 21 casos $(0,07 \%)$.

Tabla II. Consonantes iniciales más omitidas, ordenadas de mayor a menor.

\begin{tabular}{|c|c|c|c|c|c|}
\hline \multirow{2}{*}{\begin{tabular}{c} 
Consonante $\begin{array}{c}\text { Rasgos articulatorios consonante inicial dictada } \\
\text { dictada }\end{array}$ \\
\cline { 2 - 6 }
\end{tabular}} & $\begin{array}{c}\text { Lugar de } \\
\text { articulación }\end{array}$ & $\begin{array}{c}\text { Modo de } \\
\text { articulación }\end{array}$ & $\begin{array}{c}\text { Acción de } \\
\text { las cuerdas } \\
\text { vocales }\end{array}$ & $\begin{array}{c}\mathrm{N}^{\circ} \text { de } \\
\text { casos }\end{array}$ & $\%$ \\
\hline $\mathrm{p}$ & bilabial & oclusiva & sorda & 116 & $0,37 \%$ \\
\hline $\mathrm{k}$ & velar & oclusiva & sorda & 103 & $0,33 \%$ \\
\hline $\mathrm{t}$ & dental & oclusiva & sorda & 80 & $0,26 \%$ \\
\hline $\mathrm{j}$ & velar & aproximante & sorda & 75 & $0,24 \%$ \\
\hline $\mathrm{g}$ & velar & oclusiva & sonora & 54 & $0,17 \%$ \\
\hline $\mathrm{d}$ & dental & oclusiva & sonora & 41 & $0,13 \%$ \\
\hline $\mathrm{ll}$ & palatal & aproximante & sonora & 40 & $0,13 \%$ \\
\hline $\mathrm{b}$ & bilabial & oclusiva & sonora & 39 & $0,13 \%$ \\
\hline $\mathrm{f}$ & labiodental & aproximante & sorda & 35 & $0,11 \%$ \\
\hline $\mathrm{r}$ & alveolar & vibrante múltiple & sonora & 34 & $0,11 \%$ \\
\hline $\mathrm{n}$ & alveolar & nasal & sonora & 26 & $0,08 \%$ \\
\hline $\mathrm{ch}$ & palatal & africada & sorda & 24 & $0,08 \%$ \\
\hline $\mathrm{l}$ & alveolar & lateral & sonora & 22 & $0,07 \%$ \\
\hline $\mathrm{m}$ & bilabial & nasal & sonora & 21 & $0,07 \%$ \\
\hline $\mathrm{s}$ & alveolar & aproximante & sorda & 18 & $0,06 \%$ \\
\hline $\mathrm{n}$ & palatal & nasal & sonora & 10 & $0,03 \%$ \\
\hline
\end{tabular}

Las consonantes iniciales más confundidas son: la m que se confunde con la "n" (95,65\%), la "n que se confunde con " $m$ " (84,96\%), la "b" que se confunde con la "d" (63,22\%), la "ll" que se confunde con la "g" (62,07\%), la "j" que se confunde con la "g" $(57,57 \%)$, la "r" que se confunde con la "b" $(56,25 \%)$ y la " $\mathrm{t}$ " 
que se confunde con la "p" $(55,35 \%)$. Articulatoriamente, los tres primeros pares y el séptimo $(\mathrm{m} / \mathrm{n}, \mathrm{n} / \mathrm{m}, \mathrm{b} / \mathrm{d}, \mathrm{t} / \mathrm{p})$ comparten el modo y la acción de las cuerdas vocales; el cuarto y el sexto par $(11 / \mathrm{g}, \mathrm{r} / \mathrm{b})$ comparten la acción de las cuerdas vocales y el quinto par $(\mathrm{j} / \mathrm{g})$, el lugar.

Tabla III. Consonantes iniciales más confundidas, ordenadas de mayor a menor.

\begin{tabular}{|c|c|c|c|c|c|}
\hline \multirow{2}{*}{$\begin{array}{c}\text { Consonante } \\
\text { inicial } \\
\text { dictada/ } \\
\text { respondida }\end{array}$} & \multicolumn{4}{|c}{ Rasgos articulatorios consonante inicial dictada/respondida } \\
\cline { 2 - 7 } & $\begin{array}{c}\text { Lugar de } \\
\text { articulación }\end{array}$ & $\begin{array}{c}\text { Modo de } \\
\text { articulación }\end{array}$ & $\begin{array}{c}\text { Acción de } \\
\text { las cuerdas } \\
\text { vocales }\end{array}$ & $\begin{array}{c}\mathrm{N}^{\circ} \text { de } \\
\text { casos }\end{array}$ & $\%$ \\
\hline $\mathrm{m} / \mathrm{n}$ & bilabial/alveolar & nasal/nasal & sonora/sonora & 44 & $95,65 \%$ \\
\hline $\mathrm{n} / \mathrm{m}$ & alveolar/bilabial & nasal/nasal & sonora/sonora & 113 & $84,96 \%$ \\
\hline $\mathrm{b} / \mathrm{d}$ & bilabial/dental & oclusiva/oclusiva & sonora/sonora & 55 & $63,22 \%$ \\
\hline $\mathrm{ll} / \mathrm{g}$ & palatal/velar & $\begin{array}{c}\text { aproximante/ } \\
\text { oclusiva }\end{array}$ & sonora/sonora & 54 & $62,07 \%$ \\
\hline $\mathrm{j} / \mathrm{g}$ & velar/velar & $\begin{array}{c}\text { aproximante/ } \\
\text { oclusiva }\end{array}$ & sorda/sonora & 194 & $57,57 \%$ \\
\hline $\mathrm{r} / \mathrm{b}$ & alveolar/bilabial & $\begin{array}{c}\text { vibrante Múlt./ } \\
\text { oclusiva }\end{array}$ & sonora/sonora & 9 & $56,25 \%$ \\
\hline $\mathrm{t} / \mathrm{p}$ & dental/bilabial & oclusiva/oclusiva & sorda/sorda & 150 & $55,35 \%$ \\
\hline $\mathrm{ch} / \mathrm{k}$ & palatal/velar & africada/oclusiva & sorda/sorda & 11 & $44,00 \%$ \\
\hline $\mathrm{n} / \mathrm{m}$ & palatal/bilabial & nasal/nasal & sonora/sonora & 7 & $43,75 \%$ \\
\hline $\mathrm{d} / \mathrm{t}$ & dental/dental & oclusiva/oclusiva & sonora/sorda & 69 & $42,59 \%$ \\
\hline $\mathrm{k} / \mathrm{j}$ & velar/velar & $\begin{array}{c}\text { oclusiva/ } \\
\text { aproximante }\end{array}$ & sorda/sorda & 117 & $38,61 \%$ \\
\hline $\mathrm{f} / \mathrm{n}$ & alveolar/alveolar & lateral/nasal & sonora/sonora & 8 & $36,36 \%$ \\
\hline $\mathrm{g} / \mathrm{b}$ & velar/bilabial & oclusiva/oclusiva & sonora/sonora & 74 & $36,10 \%$ \\
\hline $\mathrm{s} / \mathrm{j}$ & alveolar/velar & $\begin{array}{c}\text { aproximante/ } \\
\text { aproximante }\end{array}$ & sorda/sorda & 7 & $35,00 \%$ \\
\hline & bilabial/dental & $\begin{array}{c}\text { aproximante/ } \\
\text { oclusiva }\end{array}$ & sorda/sorda & 38 & $30,65 \%$ \\
\hline & oclusiva/oclusiva & sorda/sorda & 65 & $29,02 \%$ \\
\hline
\end{tabular}

A continuación se presenta una tabla que muestra las consonantes que alcanzaron menos del $1 \%$ del total relativo de confusiones. Los pares menos confundidos son: f/ch, g/n, g/s, j/l, j/ch, k/b, k/d, n/d, n/j, n/t, p/m, p/n, t/b, t/ch, t/n, t/ll, de los cuales, 9 comparten un solo rasgo articulatorio: la acción de las cuerdas vocales (f/ch, g/n, j/ch, n/d, t/ch), el modo (k/b, k/d, t/b) y el lugar (p/m) y 7 no comparten ningún rasgo articulatorio: $(\mathrm{g} / \mathrm{s}, \mathrm{j} / \mathrm{l}, \mathrm{n} / \mathrm{j}, \mathrm{n} / \mathrm{t}, \mathrm{p} / \mathrm{n}, \mathrm{t} / \mathrm{n}, \mathrm{t} / \mathrm{ll})$. 
Tabla IV. Consonantes iniciales menos confundidas

\begin{tabular}{|c|c|c|c|c|c|}
\hline \multirow[b]{2}{*}{$\begin{array}{l}\text { Consonante } \\
\text { inicial } \\
\text { dictada/ } \\
\text { respondida }\end{array}$} & \multicolumn{5}{|c|}{ Rasgos articulatorios de la consonante inicial dictada/respondida } \\
\hline & $\begin{array}{c}\text { Lugar de } \\
\text { articulación }\end{array}$ & $\begin{array}{c}\text { Modo de } \\
\text { articulación }\end{array}$ & $\begin{array}{c}\text { Acción } \\
\text { de las cuerdas } \\
\text { vocales }\end{array}$ & $\begin{array}{l}\mathrm{N}^{\circ} \text { de } \\
\text { casos }\end{array}$ & $\%$ \\
\hline $\mathrm{f} / \mathrm{ch}$ & $\begin{array}{l}\text { labiodental/ } \\
\text { palatal }\end{array}$ & $\begin{array}{l}\text { aproximante/ } \\
\text { africada }\end{array}$ & sorda/sorda & 1 & $0,81 \%$ \\
\hline $\mathrm{g} / \mathrm{n}$ & velar/alveolar & oclusiva/nasal & sonora/sonora & 1 & $0,49 \%$ \\
\hline $\mathrm{g} / \mathrm{s}$ & velar/alveolar & $\begin{array}{c}\text { oclusiva/ } \\
\text { aproximante }\end{array}$ & sonora/sorda & 1 & $0,49 \%$ \\
\hline $\mathrm{j} / \mathrm{l}$ & velar/alveolar & $\begin{array}{c}\text { aproximante/ } \\
\text { lateral }\end{array}$ & sorda/sonora & 2 & $0,59 \%$ \\
\hline $\mathrm{j} / \mathrm{ch}$ & velar/palatal & $\begin{array}{l}\text { aproximantel } \\
\text { africada }\end{array}$ & sorda/sorda & 1 & $0,30 \%$ \\
\hline $\mathrm{k} / \mathrm{b}$ & velar/bilabial & oclusiva/oclusiva & sorda/sonora & 1 & $0,33 \%$ \\
\hline $\mathrm{k} / \mathrm{d}$ & velar/dental & oclusiva/oclusiva & sorda/sonora & 1 & $0,33 \%$ \\
\hline $\mathrm{n} / \mathrm{d}$ & alveolar/dental & nasal/oclusiva & sonora/sonora & 1 & $0,75 \%$ \\
\hline $\mathrm{n} / \mathrm{j}$ & alveolar/velar & $\begin{array}{c}\text { nasal/ } \\
\text { aproximante }\end{array}$ & sonora/sorda & 1 & $0,75 \%$ \\
\hline $\mathrm{n} / \mathrm{t}$ & alveolar/dental & nasal/oclusiva & sonora/sorda & 1 & $0,75 \%$ \\
\hline $\mathrm{p} / \mathrm{m}$ & bilabial/bilabial & oclusiva/nasal & sorda/sonora & 1 & $0,45 \%$ \\
\hline $\mathrm{p} / \mathrm{n}$ & bilabial/alveolar & oclusiva/nasal & sorda/sonora & 1 & $0,45 \%$ \\
\hline $\mathrm{t} / \mathrm{b}$ & dental/bilabial & oclusiva/oclusiva & sorda/sonora & 2 & $0,74 \%$ \\
\hline $\mathrm{t} / \mathrm{ch}$ & dental/palatal & oclusiva/africada & sorda/sorda & 1 & $0,37 \%$ \\
\hline $\mathrm{t} / \mathrm{n}$ & dental/alveolar & oclusiva/nasal & sorda/sonora & 1 & $0,37 \%$ \\
\hline$t / l l$ & dental/palatal & $\begin{array}{c}\text { oclusiva/ } \\
\text { aproximante }\end{array}$ & sorda/sonora & 1 & $0,37 \%$ \\
\hline
\end{tabular}

Las consonantes que aparecen encabezando el mayor número de confusiones de más de una consonante en posición inicial son: las oclusivas $\mathrm{p}(\mathrm{t} / \mathrm{p}, \mathrm{f} / \mathrm{p}), \mathrm{b}(\mathrm{r} / \mathrm{b}$, $\mathrm{g} / \mathrm{b}), \mathrm{t}(\mathrm{d} / \mathrm{t}, \mathrm{p} / \mathrm{t}), \mathrm{g}(\mathrm{ll} / \mathrm{g}, \mathrm{j} / \mathrm{g})(2$ veces $)$, las nasales $\mathrm{m}(\mathrm{n} / \mathrm{m}, \tilde{\mathrm{n}} / \mathrm{m})$ y n $(\mathrm{m} / \mathrm{n}, \mathrm{l} / \mathrm{n})(2$ veces) y la aproximante $\mathrm{j}(\mathrm{k} / \mathrm{j}, \mathrm{s} / \mathrm{j})(2$ veces $)$.

A continuación se presenta un cuadro cuyo eje vertical contiene las consonantes articuladas y el eje horizontal, las consonantes erradas. 
Tabla V. Confusión de la consonante inicial.

\begin{tabular}{|c|c|c|c|c|c|c|c|c|c|c|c|c|c|c|c|c|}
\hline \multirow{2}{*}{$\begin{array}{l}\text { Consonante } \\
\text { inicial } \\
\text { respondida }\end{array}$} & \multicolumn{16}{|c|}{ Consonante inicial (dictada) } \\
\hline & $\mathrm{p}$ & b & $\mathrm{m}$ & $\mathrm{f}$ & $\mathrm{t}$ & d & s & $\mathrm{n}$ & 1 & $\mathrm{r}$ & ch & Il & $\tilde{\mathbf{n}}$ & $\mathrm{k}$ & $\mathrm{g}$ & j \\
\hline $\mathrm{p}$ & & & & & $\mathrm{x}$ & & & & & & & & & & & \\
\hline $\mathrm{b}$ & & & & & & $\mathrm{x}$ & & & & & & & & & & \\
\hline $\mathrm{m}$ & & & & & & & & $\mathrm{x}$ & & & & & & & & \\
\hline $\mathrm{f}$ & $\mathrm{x}$ & & & & & & & & & & & & & & & \\
\hline $\mathrm{t}$ & $\mathrm{x}$ & & & & & & & & & & & & & & & \\
\hline d & & & & & $\mathrm{x}$ & & & & & & & & & & & \\
\hline s & & & & & & & & & & & & & & & & $\mathrm{x}$ \\
\hline $\mathrm{n}$ & & & $\mathrm{x}$ & & & & & & & & & & & & & \\
\hline 1 & & & & & & & & $\mathrm{x}$ & & & & & & & & \\
\hline $\mathrm{r}$ & & $\mathrm{x}$ & & & & & & & & & & & & & & \\
\hline $\mathrm{ch}$ & & & & & & & & & & & & & & $\mathrm{x}$ & & \\
\hline 11 & & & & & & & & & & & & & & & $\mathrm{x}$ & \\
\hline$\tilde{\mathrm{n}}$ & & & $\mathrm{x}$ & & & & & & & & & & & & & \\
\hline $\mathrm{k}$ & & & & & & & & & & & & & & & & $\mathrm{x}$ \\
\hline $\mathrm{g}$ & & $\mathrm{x}$ & & & & & & & & & & & & & & \\
\hline $\mathrm{j}$ & & & & & & & & & & & & & & & $\mathrm{x}$ & \\
\hline Totales & 2 & 2 & 2 & 0 & 2 & 1 & 0 & 2 & 0 & 0 & 0 & 0 & 0 & 1 & 2 & 2 \\
\hline
\end{tabular}

\subsubsection{La vocal}

La vocal al igual que la consonante en posición final no presentó casos de omisión. Las vocales que más se confunden en más de un $50 \%$ de los casos en que aparecen dentro de la combinación consonante + vocal + consonante son: la "e" que se confunde con la "a", sin rasgos comunes entre sí; la "i" que se confunde con la "e", anteriores; la "o" que se confunde con la "u", posteriores; y la "a" que se confunde con la "o", sin rasgos comunes, en ese orden. En el extremo opuesto, los pares de vocales que presentan menos de un 10\% de confusión entre sí son: la "e" con la "i", anteriores; la "u" con la "e", sin rasgos comunes; la "u" con la "a", sin rasgos comunes; la "o" con la "i", sin rasgos comunes; la "o" con la "e", medias; la "a" con la "i", sin rasgos comunes; la "e" con la "u", sin rasgos comunes; la "e" con la "o", medias. 
Tabla VI. Cuadro general de vocales confundidas, ordenadas de mayor a menor.

\begin{tabular}{|c|c|c|c|c|}
\hline \multirow{2}{*}{$\begin{array}{c}\text { Vocal dictada/ } \\
\text { respondida }\end{array}$} & \multicolumn{4}{|c|}{ Rasgos articulatorios de la vocal dictada/respondida } \\
\cline { 2 - 5 } & $\begin{array}{c}\text { Lugar de } \\
\text { articulación }\end{array}$ & $\begin{array}{c}\text { Modo de } \\
\text { articulación }\end{array}$ & $\begin{array}{c}\mathrm{N}^{\circ} \text { de } \\
\text { casos }\end{array}$ & $\%$ \\
\hline $\mathrm{e} / \mathrm{a}$ & anterior/central & media/baja & 19 & $79,17 \%$ \\
\hline $\mathrm{i} / \mathrm{e}$ & anterior/anterior & alta/media & 7 & $77,78 \%$ \\
\hline $\mathrm{o} / \mathrm{u}$ & posterior/posterior & media/alta & 31 & $77,50 \%$ \\
\hline $\mathrm{a} / \mathrm{o}$ & central/posterior & baja/media & 9 & $60,00 \%$ \\
\hline $\mathrm{u} / \mathrm{i}$ & posterior/anterior & alta/alta & 9 & $45,00 \%$ \\
\hline $\mathrm{u} / \mathrm{o}$ & posterior/posterior & alta/media & 9 & $45,00 \%$ \\
\hline $\mathrm{a} / \mathrm{e}$ & central/anterior & baja/media & 5 & $33,33 \%$ \\
\hline $\mathrm{i} / \mathrm{u}$ & anterior/posterior & alta/alta & 2 & $22,22 \%$ \\
\hline $\mathrm{o} / \mathrm{a}$ & posterior/central & media/baja & 5 & $12,50 \%$ \\
\hline $\mathrm{e} / \mathrm{o}$ & anterior/posterior & media/media & 2 & $8,33 \%$ \\
\hline $\mathrm{e} / \mathrm{u}$ & anterior/posterior & media/alta & 2 & $8,33 \%$ \\
\hline $\mathrm{a} / \mathrm{i}$ & central/anterior & baja/alta & 1 & $6,67 \%$ \\
\hline $\mathrm{o} / \mathrm{e}$ & posterior/anterior & media/media & 2 & $5,00 \%$ \\
\hline $\mathrm{o} / \mathrm{i}$ & posterior/anterior & media/alta & 2 & $5,00 \%$ \\
\hline $\mathrm{u} / \mathrm{a}$ & posterior/central & alta/baja & 1 & $5,00 \%$ \\
\hline $\mathrm{u} / \mathrm{e}$ & posterior/anterior & alta/media & 1 & $5,00 \%$ \\
\hline $\mathrm{e} / \mathrm{i}$ & anterior/anterior & media/alta & 1 & $4,17 \%$ \\
\hline
\end{tabular}

\subsubsection{La consonante final}

La consonante final tal como la vocal interior no presentó casos de omisión. Las consonantes finales más confundidas son: la " $m$ " que se confunde con la " $n$ " $(93,03 \%)$, la "n" que se confunde con la "m" $(88,53 \%)$, la "j" que se confunde con la "g" $(73,90 \%)$, la " $k$ " que se confunde con la "g" $(63,64 \%)$, y la "t" que se confunde con la "d" (57,90\%).

Se desprende que los pares que alcanzan $50 \%$ o más coinciden en el modo y la acción de las cuerdas vocales $(\mathrm{m} / \mathrm{n}, \mathrm{n} / \mathrm{m})$, el lugar y el modo ( $\mathrm{j} / \mathrm{g}$ ) y el lugar solo $(\mathrm{k} / \mathrm{g}, \mathrm{t} / \mathrm{d})$. 
Tabla VII. Consonantes finales más confundidas, ordenadas de mayor a menor.

\begin{tabular}{|c|c|c|c|c|c|}
\hline \multirow[b]{2}{*}{$\begin{array}{l}\text { Consonante final } \\
\text { dictada/respondida }\end{array}$} & \multicolumn{5}{|c|}{ Rasgos articulatorios de la consonante final dictada/confundida } \\
\hline & $\begin{array}{l}\text { Lugar de } \\
\text { articulación }\end{array}$ & $\begin{array}{l}\text { Modo de } \\
\text { articulación }\end{array}$ & $\begin{array}{c}\text { Acción de } \\
\text { las cuerdas } \\
\text { vocales }\end{array}$ & $\begin{array}{l}\mathrm{N}^{\circ} \text { de } \\
\text { casos }\end{array}$ & $\%$ \\
\hline $\mathrm{m} / \mathrm{n}$ & bilabial/alveolar & nasal/nasal & $\begin{array}{c}\text { sonora/ } \\
\text { sonora }\end{array}$ & 267 & $93,03 \%$ \\
\hline $\mathrm{n} / \mathrm{m}$ & alveolar/bilabial & nasal/nasal & $\begin{array}{l}\text { sonora/ } \\
\text { sonora }\end{array}$ & 247 & $88,53 \%$ \\
\hline$j / g$ & velar/velar & $\begin{array}{l}\text { aproximante/ } \\
\text { aproximante }\end{array}$ & $\begin{array}{l}\text { sorda/s } \\
\text { onora }\end{array}$ & 354 & $73,90 \%$ \\
\hline $\mathrm{k} / \mathrm{g}$ & velar/velar & $\begin{array}{c}\text { oclusiva/ } \\
\text { aproximante }\end{array}$ & $\begin{array}{l}\text { sorda/ } \\
\text { sonora }\end{array}$ & 42 & $63,64 \%$ \\
\hline $\mathrm{t} / \mathrm{d}$ & dental/dental & $\begin{array}{c}\text { oclusiva/ } \\
\text { aproximante }\end{array}$ & $\begin{array}{l}\text { sorda/ } \\
\text { sonora }\end{array}$ & 132 & $57,90 \%$ \\
\hline $1 / \mathrm{k}$ & alveolar/velar & lateral/oclusiva & $\begin{array}{l}\text { sonora/ } \\
\text { sorda }\end{array}$ & 6 & $46,15 \%$ \\
\hline $\mathrm{g} / \mathrm{b}$ & velar/bilabial & $\begin{array}{l}\text { aproximante/ } \\
\text { aproximante }\end{array}$ & $\begin{array}{c}\text { sonora/ } \\
\text { sonora }\end{array}$ & 214 & $40,61 \%$ \\
\hline$d / t$ & dental/dental & $\begin{array}{l}\text { aproximante/ } \\
\text { oclusiva }\end{array}$ & $\begin{array}{c}\text { sonora/ } \\
\text { sorda }\end{array}$ & 314 & $39,65 \%$ \\
\hline $\mathrm{p} / \mathrm{b}$ & bilabial/bilabial & $\begin{array}{c}\text { oclusiva/ } \\
\text { aproximante }\end{array}$ & $\begin{array}{l}\text { sorda/ } \\
\text { sonora }\end{array}$ & 306 & $38,88 \%$ \\
\hline $\mathrm{b} / \mathrm{d}$ & bilabial/dental & $\begin{array}{l}\text { aproximante/ } \\
\text { aproximante }\end{array}$ & $\begin{array}{c}\text { sonora/ } \\
\text { sonora }\end{array}$ & 231 & $37,81 \%$ \\
\hline $\mathrm{r} / \mathrm{g}$ & alveolar/velar & $\begin{array}{c}\text { vibrante múlt./ } \\
\text { aproximante }\end{array}$ & $\begin{array}{c}\text { sonora/ } \\
\text { sonora }\end{array}$ & 26 & $35,62 \%$ \\
\hline$f / b$ & $\begin{array}{c}\text { labiodental/ } \\
\text { bilabial }\end{array}$ & $\begin{array}{l}\text { aproximante/ } \\
\text { aproximante }\end{array}$ & $\begin{array}{l}\text { sorda/ } \\
\text { sonora }\end{array}$ & 17 & $20,00 \%$ \\
\hline
\end{tabular}

Conviene consignar que en esta posición las consonantes "b", "d" y "g" se realizaron como aproximantes.

Las consonantes finales que presentan menos confusiones con otra consonante, porque representan menos del $1 \%$ de su total relativo son: $\mathrm{d} / \mathrm{j}, \mathrm{p} / \mathrm{l}, \mathrm{p} / \mathrm{o}, \mathrm{p} / \mathrm{s}, \mathrm{g} / \mathrm{i}$, $\mathrm{j} / \mathrm{l}, \mathrm{j} / \mathrm{n}, \mathrm{b} / \mathrm{k}, \mathrm{m} / \mathrm{j}, \mathrm{n} / \mathrm{b}, \mathrm{n} / \mathrm{d}, \mathrm{g} / \mathrm{f}, \mathrm{j} / \mathrm{s}, \mathrm{t} / \mathrm{s}, \mathrm{p} / \mathrm{j}, \mathrm{m} / \mathrm{l}, \mathrm{m} / \mathrm{p}, \mathrm{m} / \mathrm{r}, \mathrm{n} / \mathrm{r}, \mathrm{g} / \mathrm{l}, \mathrm{g} / \mathrm{m}, \mathrm{b} / \mathrm{f}$.

De los 22 casos con menos del 1\%, 10 pares comparten la acción de las cuerdas vocales $(\mathrm{p} / \mathrm{s}, \mathrm{g} / \mathrm{i}, \mathrm{n} / \mathrm{b}, \mathrm{n} / \mathrm{d}, \mathrm{t} / \mathrm{s}, \mathrm{p} / \mathrm{j}, \mathrm{m} / \mathrm{r}, \mathrm{n} / \mathrm{r}, \mathrm{g} / \mathrm{l}, \mathrm{g} / \mathrm{m}) ; 3$ pares comparten el modo fricativo $(\mathrm{d} / \mathrm{j}, \mathrm{g} / \mathrm{f}, \mathrm{b} / \mathrm{f}) ; 1$ par comparte el lugar bilabial $(\mathrm{m} / \mathrm{p}) ; 1$ par comparte el modo fricativo y la sordez $(\mathrm{j} / \mathrm{s})$; y los 7 pares restantes no comparten nada $(\mathrm{p} / \mathrm{l}$, $\mathrm{p} / \mathrm{o}, \mathrm{j} / \mathrm{l}, \mathrm{j} / \mathrm{n}, \mathrm{b} / \mathrm{k}, \mathrm{m} / \mathrm{j}, \mathrm{m} / \mathrm{l})$. 
Tabla VIII. Consonantes finales menos confundidas, ordenadas de menor a mayor.

\begin{tabular}{|c|c|c|c|c|c|}
\hline \multirow{2}{*}{$\begin{array}{l}\text { Consonante } \\
\text { final dictada/ } \\
\text { respondida }\end{array}$} & \multicolumn{5}{|c|}{ Rasgos articulatorios de la consonante final dictada/respondida } \\
\hline & $\begin{array}{c}\text { Lugar de } \\
\text { articulación }\end{array}$ & $\begin{array}{l}\text { Modo de } \\
\text { articulación }\end{array}$ & $\begin{array}{l}\text { Acción de las } \\
\text { cuerdas vocales }\end{array}$ & $\begin{array}{l}\mathrm{N}^{\circ} \text { de } \\
\text { casos }\end{array}$ & $\%$ \\
\hline$d / j$ & dental/velar & $\begin{array}{l}\text { aproximante/ } \\
\text { aproximante }\end{array}$ & sonora/sorda & 1 & $0,13 \%$ \\
\hline $\mathrm{p} / \mathrm{l}$ & bilabial/alveolar & oclusiva/lateral & sorda/sonora & 1 & $0,13 \%$ \\
\hline $\mathrm{p} / \mathrm{o}$ & bilabial/posterior & oclusiva/media & sorda/sonora & 1 & $0,13 \%$ \\
\hline $\mathrm{p} / \mathrm{s}$ & bilabial/alveolar & $\begin{array}{c}\text { oclusiva/ } \\
\text { aproximante }\end{array}$ & sorda/sorda & 1 & $0,13 \%$ \\
\hline$g / i$ & velar/anterior & aproximante/alta & sonora/sonora & 1 & $0,19 \%$ \\
\hline $\mathrm{j} / \mathrm{l}$ & velar/alveolar & $\begin{array}{c}\text { aproximante/ } \\
\text { lateral }\end{array}$ & sorda/sonora & 1 & $0,21 \%$ \\
\hline $\mathrm{j} / \mathrm{n}$ & velar/alveolar & $\begin{array}{c}\text { aproximante/ } \\
\text { nasal }\end{array}$ & sorda/sonora & 1 & $0,21 \%$ \\
\hline $\mathrm{b} / \mathrm{k}$ & bilabial/velar & $\begin{array}{l}\text { aproximante/ } \\
\text { oclusiva }\end{array}$ & sonora/sorda & 2 & $0,33 \%$ \\
\hline $\mathrm{m} / \mathrm{j}$ & bilabial/velar & $\begin{array}{c}\text { nasal/ } \\
\text { aproximante }\end{array}$ & sonora/sorda & 1 & $0,35 \%$ \\
\hline $\mathrm{n} / \mathrm{b}$ & alveolar/bilabial & $\begin{array}{c}\text { nasal/ } \\
\text { aproximante }\end{array}$ & sonora/sonora & 1 & $0,36 \%$ \\
\hline $\mathrm{n} / \mathrm{d}$ & alveolar/dental & $\begin{array}{c}\text { nasal/ } \\
\text { aproximante }\end{array}$ & sonora/sonora & 1 & $0,36 \%$ \\
\hline$g / f$ & velar/labiodental & $\begin{array}{l}\text { aproximante/ } \\
\text { aproximante }\end{array}$ & sonora/sorda & 2 & $0,38 \%$ \\
\hline$j / s$ & velar/alveolar & $\begin{array}{l}\text { aproximante/ } \\
\text { aproximante }\end{array}$ & sorda/sorda & 2 & $0,42 \%$ \\
\hline $\mathrm{t} / \mathrm{s}$ & dental/alveolar & $\begin{array}{c}\text { oclusiva/ } \\
\text { aproximante }\end{array}$ & sorda/sorda & 1 & $0,44 \%$ \\
\hline $\mathrm{p} / \mathrm{j}$ & bilabial/velar & $\begin{array}{c}\text { oclusiva/ } \\
\text { aproximante }\end{array}$ & sorda/sorda & 4 & $0,51 \%$ \\
\hline $\mathrm{m} / \mathrm{l}$ & bilabial/alveolar & nasal/lateral & sonora/sonora & 2 & $0,70 \%$ \\
\hline $\mathrm{m} / \mathrm{p}$ & bilabial/bilabial & nasal/oclusiva & sonora/sorda & 2 & $0,70 \%$ \\
\hline $\mathrm{m} / \mathrm{r}$ & bilabial/alveolar & nasal/vibr. múlt. & sonora/sonora & 2 & $0,70 \%$ \\
\hline $\mathrm{n} / \mathrm{r}$ & alveolar/alveolar & nasal/ vibr. múlt. & sonora/sonora & 2 & $0,717 \%$ \\
\hline$g / 1$ & velar/alveolar & $\begin{array}{c}\text { aproximante/ } \\
\text { lateral }\end{array}$ & sonora/sonora & 4 & $0,76 \%$ \\
\hline $\mathrm{g} / \mathrm{m}$ & velar/bilabial & $\begin{array}{c}\text { aproximante/ } \\
\text { nasal }\end{array}$ & sonora/sonora & 5 & $0,95 \%$ \\
\hline $\mathrm{b} / \mathrm{f}$ & bilabial/labiodental & $\begin{array}{l}\text { aproximante/ } \\
\text { aproximante }\end{array}$ & sonora/sorda & 6 & $0,98 \%$ \\
\hline
\end{tabular}


Las consonantes que aparecen encabezando el mayor número de confusiones de más de una consonante en posición final son las aproximantes sonoras "b", "g", seguidas de la aproximante sonora "d".

No figuran dentro de las más confundidas la bilabial oclusiva sorda "p", la labiodental aproximante sorda "f", la alveolar lateral sonora "l", la alveolar vibrante múltiple sonora " $r$ ", la velar aproximante sorda "j".

Téngase en cuenta que las consonantes que no integran el repertorio de las 12 consonantes finales $(t, s, c h, 1 l, \tilde{n})$, tampoco aparecen dentro de las opciones con las que los auditores confunden consonantes; en cambio aparecen las vocales "o" (en lugar de "p", un caso), "i" (en lugar de "g", un caso), a pesar de que los auditores habían sido informados de que la estructura de la palabra escuchada sería CVC.

A continuación se presenta un cuadro cuyo eje vertical contiene las consonantes articuladas y el eje horizontal, las consonantes erradas.

Tabla IX. Confusión de la consonante final.

\begin{tabular}{|c|c|c|c|c|c|c|c|c|c|c|c|c|}
\hline \multirow{2}{*}{$\begin{array}{c}\text { Consonante } \\
\text { final respondida }\end{array}$} & \multicolumn{9}{|c|}{ Consonante final dictada } \\
\hline & $\mathrm{p}$ & $\mathrm{b}$ & $\mathrm{m}$ & $\mathrm{f}$ & $\mathrm{t}$ & $\mathrm{d}$ & $\mathrm{n}$ & $\mathrm{l}$ & $\mathrm{r}$ & $\mathrm{k}$ & $\mathrm{g}$ & $\mathrm{j}$ \\
\hline $\mathrm{p}$ & & $\mathrm{x}$ & & & & & & & & & & \\
\hline $\mathrm{b}$ & & & & & & $\mathrm{x}$ & & & & & & \\
\hline $\mathrm{m}$ & & & & & & & $\mathrm{x}$ & & & & & \\
\hline $\mathrm{f}$ & & $\mathrm{x}$ & & & & & & & & & & \\
\hline $\mathrm{t}$ & & & & & & $\mathrm{x}$ & & & & & & \\
\hline $\mathrm{d}$ & & & & & $\mathrm{x}$ & & & & & & & \\
\hline $\mathrm{n}$ & & & $\mathrm{x}$ & & & & & & & & & \\
\hline $\mathrm{l}$ & & & & & & & & & & $\mathrm{x}$ & & \\
\hline $\mathrm{r}$ & & & & & & & & & & & $\mathrm{x}$ & \\
\hline $\mathrm{k}$ & & & & & & & & & & & $\mathrm{x}$ & \\
\hline $\mathrm{g}$ & & $\mathrm{x}$ & & & & & & & & & & \\
\hline $\mathrm{j}$ & & & & & & & & & & & $\mathrm{x}$ & \\
\hline Totales & 0 & 3 & 1 & 0 & 1 & 2 & 1 & 0 & 0 & 1 & 3 & 0 \\
\hline
\end{tabular}




\subsubsection{Comparación de la consonante inicial y la consonante final en la estructura CVC}

También se observa que 6 consonantes de las 12 que están presentes en la posición inicial y final de los logatomos articulados (p, b, m, f, t, d, n, l, r, k, g, j) de estructura CVC coinciden en la consonante con la que más se confundieron, a saber: "b" con "d", "m" con "n", "t" con "d", "n" con "m", "g" con "b", "j" con "g”.

Tabla X. Cuadro comparativo de las confusiones más frecuentes de las consonantes iniciales y finales.

\begin{tabular}{|c|c|c|c|c|c|c|c|c|c|}
\hline \multicolumn{5}{|c|}{ Consonante inicial } & \multicolumn{5}{|c|}{ Consonante final } \\
\hline $\begin{array}{c}\mathrm{Se} \\
\text { articula }\end{array}$ & $\begin{array}{c}\text { Se } \\
\text { escribe }\end{array}$ & $\begin{array}{c}\text { Lugar de } \\
\text { articulación }\end{array}$ & $\begin{array}{c}\text { Modo de } \\
\text { articulación }\end{array}$ & $\begin{array}{c}\text { Acción } \\
\text { de las } \\
\text { cuerdas } \\
\text { vocales }\end{array}$ & $\begin{array}{c}\mathrm{Se} \\
\text { articula }\end{array}$ & $\begin{array}{c}\mathrm{Se} \\
\text { escribe }\end{array}$ & $\begin{array}{c}\text { Lugar de } \\
\text { articulación }\end{array}$ & $\begin{array}{c}\text { Modo de } \\
\text { articulación }\end{array}$ & $\begin{array}{c}\text { Acción } \\
\text { de las } \\
\text { cuerdas } \\
\text { vocales }\end{array}$ \\
\hline $\mathrm{P}$ & $\mathrm{t}$ & & $\mathrm{x}$ & $\mathrm{x}$ & $\mathrm{p}$ & $\mathrm{b}$ & $\mathrm{x}$ & & \\
\hline $\mathrm{b}$ & $\mathrm{d}$ & & $\mathrm{x}$ & $\mathrm{x}$ & $\mathrm{b}$ & d & & $\mathrm{x}$ & $\mathrm{x}$ \\
\hline $\mathrm{m}$ & $\mathrm{n}$ & & $\mathrm{x}$ & $\mathrm{x}$ & $\mathrm{m}$ & $\mathrm{n}$ & & $\mathrm{x}$ & $\mathrm{x}$ \\
\hline $\mathrm{f}$ & $\mathrm{p}$ & & & $\mathrm{x}$ & $\mathrm{f}$ & $\mathrm{b}$ & & $\mathrm{x}$ & \\
\hline $\mathrm{t}$ & $\mathrm{p}$ & & $\mathrm{x}$ & $\mathrm{x}$ & $\mathrm{t}$ & $\mathrm{d}$ & $\mathrm{x}$ & & \\
\hline d & $\mathrm{t}$ & $\mathrm{x}$ & $\mathrm{x}$ & & $\mathrm{d}$ & $\mathrm{t}$ & $\mathrm{x}$ & & \\
\hline s & j & & $\mathrm{x}$ & $\mathrm{x}$ & & & & & \\
\hline $\mathrm{n}$ & $\mathrm{m}$ & & $\mathrm{x}$ & $\mathrm{x}$ & $\mathrm{n}$ & $\mathrm{m}$ & & $\mathrm{x}$ & $\mathrm{x}$ \\
\hline 1 & $\mathrm{n}$ & $\mathrm{x}$ & & $\mathrm{x}$ & 1 & $\mathrm{k}$ & & & \\
\hline $\mathrm{r}$ & $\mathrm{b}$ & & & $\mathrm{x}$ & $\mathrm{r}$ & g & & & $\mathrm{x}$ \\
\hline $\mathrm{ch}$ & $\mathrm{k}$ & & & $\mathrm{x}$ & & & & & \\
\hline 11 & $\mathrm{~g}$ & & & $\mathrm{x}$ & & & & & \\
\hline$\tilde{\mathbf{n}}$ & $\mathrm{m}$ & & $\mathrm{x}$ & $\mathrm{x}$ & & & & & \\
\hline $\mathrm{k}$ & j & $\mathrm{x}$ & & $\mathrm{x}$ & $\mathrm{k}$ & g & $\mathrm{x}$ & & \\
\hline $\mathrm{g}$ & $\mathrm{b}$ & & $\mathrm{x}$ & $\mathrm{x}$ & $\mathrm{g}$ & $\mathrm{b}$ & & $\mathrm{x}$ & $\mathrm{x}$ \\
\hline j & $\mathrm{g}$ & $\mathrm{x}$ & & & j & $\mathrm{g}$ & $\mathrm{x}$ & $\mathrm{x}$ & \\
\hline
\end{tabular}


Tabla XI. Cuadro de rasgos articulatorios comunes entre los pares más frecuentes de consonantes confundidas en posición inicial y final.

\begin{tabular}{|l|c|c|}
\hline \multicolumn{1}{|c|}{$\begin{array}{c}\text { Rasgos más comunes entre consonante articulada } \\
\text { y consonante errónea }\end{array}$} & $\begin{array}{c}\text { Consonante } \\
\text { inicial }\end{array}$ & $\begin{array}{c}\text { Consonante } \\
\text { final }\end{array}$ \\
\hline Lugar de articulación & 1 & 4 \\
\hline Modo de articulación & 4 & 1 \\
\hline Acción de las cuerdas vocales & 1 & 1 \\
\hline Lugar y modo de articulación & 2 & 1 \\
\hline Lugar de articulación y acción de las cuerdas vocales & 8 & 4 \\
\hline Modo de articulación y acción de las cuerdas vocales & 16 & 12 \\
\hline Total de consonantes (en posición inicial y final) & & \\
\hline
\end{tabular}

En la posición inicial, en la mitad de los casos, la consonante dictada y la consonante respondida erróneamente comparten conjuntamente el modo de articulación y la acción de las cuerdas vocales. En la posición final, la consonante dictada y la consonante errada comparten el lugar de articulación y la unión del modo de articulación y la acción de las cuerdas vocales.

\section{DISCUSIÓN Y CONCLUSIONES}

Tras este estudio hemos comprobado que en un ambiente de STI que va del 0,4 al 0,8 los auditores han fallado u omitido sus respuestas en un 25,18 \% de los casos. El análisis nos revela algunas cuestiones ya observadas en estudios anteriores, especialmente para el inglés, y otras cuestiones nuevas.

En general, podríamos decir que el ruido afecta de manera desigual a los componentes de la estructura del logatomo $\mathrm{CVC}$, al tipo de sonido consonántico o vocálico, y también, al tipo de sonido con el que se confunde cada vocal y cada consonante en particular. En el primer sentido, se producen menos errores por confusión en las vocales que en las consonantes, y entre consonantes, menos en la inicial que en la final; en otras palabras, las vocales son más resistentes al ruido que las consonantes y las consonantes iniciales son más robustas que las consonantes finales. Esto ya había sido puesto de manifiesto por Peutz (1971) y confirmado por Rosas y Sommerhoff (2008).

En el segundo sentido, las consonantes iniciales manifiestan una tendencia clara a confundirse con aquellas consonantes con las que comparten en forma conjunta el modo y la acción de las cuerdas vocales, mientras que las consonantes 
finales lo hacen por partes iguales con aquellas consonantes con las que comparten combinadamente el modo y la acción de las cuerdas vocales, pero también, cuando el único rasgo compartido es solo el lugar de articulación, haciendo sugerir que en posición inicial se requiere de más rasgos articulatorios comunes para que la confusión entre consonantes se manifieste, mientras que en posición final la tendencia a la presencia de rasgos combinados menos acentuada permite deducir la tendencia contraria; estos hechos podrían interpretarse igualmente en la línea de la mayor robustez de las consonantes iniciales frente a las consonantes finales, antes mencionada.

Desde otra perspectiva, las consonantes que aparecen dentro de las opciones más recurrentes en las confusiones de la consonante inicial y final consignadas evidencian más diversidad en la primera consonante que en la segunda (modo oclusivo, nasal y fricativo en la consonante inicial frente a fricativo solo en la consonante final), lo que podría ser una indicación de la mayor precisión requerida para las confusiones en posición inicial frente a la posición final.

Por otra parte, el hecho de que la mitad de las consonantes presentes en posición inicial y final del listado de logatomos se confundan en ambas posiciones con las mismas consonantes y que en general coincidan en el modo de articulación como característica común estaría revelando un grado de resistencia de este rasgo fonético frente al lugar y las acción de las cuerdas vocales; es decir, mayor poder distinguidor en condiciones precarias de inteligibilidad. Estos resultados coinciden con los obtenidos en estudios sobre el inglés en Ziegler et al. (2009) y sobre el español, reportado en Marrero et al. (2015).

También encuentra respaldo parcial en estudios anteriores el que las consonantes iniciales más confundidas sean las nasales y las oclusivas por concordar con los obtenidos por Parikh y Loizou (2005) para el inglés, que muestran que las oclusivas presentaron unas tasas de identificación altas incluso en condiciones extremas, donde la información sobre la barra de explosión no se preservaba.

Por su parte, aunque las vocales registran un porcentaje muy bajo de errores con solo un $0,35 \%$ del total, nos permite observar que la naturaleza de las confusiones se ve motivada, al menos, en dos de los cuatro pares que alcanzan más de un $50 \%$ de las ocurrencias, por la presencia del lugar de articulación; en tanto que la menor confusión con menos de un $10 \%$ de ocurrencias se caracteriza por no exhibir la presencia de rasgo común alguno en la mayoría de los miembros de los pares entre sí.

Aparte de estas generalidades, llama la atención el hecho de que las consonantes iniciales concentren todas las omisiones, a la vez que registran un bajo porcentaje de confusiones, y que en su mayoría correspondan a oclusivas ( $\mathrm{p}, \mathrm{k}, \mathrm{t}$, g) caracterizadas por su fuerte resistencia al ruido. Una explicación posible para esta dicotomía podría relacionarse con un problema de concentración inicial y de focalización en la imagen acústica o recuerdo del logatomo anterior que precedía 
en el dictado y no a una dificultad intrínseca de la consonante en sí. En cuanto al carácter oclusivo de las omitidas, no podemos establecer nada y tampoco encontramos estudios que mencionen el hecho.

Otra particularidad observada tiene que ver con la falta de reciprocidad en las confusiones que se da en el par i/e, donde el primer miembro se confunde en más de un $50 \%$ de los casos con la e, pero esto no ocurre a la inversa. Esta falta de reciprocidad también se da para algunas consonantes en posición inicial y final, pero de manera menos marcada. Para este hecho, tampoco hemos encontrado explicación o antecedentes.

Los resultados expuestos y las interpretaciones posibles nos sugieren caminos de investigación para develar la lógica que subyace al reconocimiento auditivo desde la perspectiva de los rasgos articulatorios de los sonidos, pero donde seguramente contribuyen otros factores que un estudio más completo tendrá que incorporar. El interés por descubrir qué hace que algunos sonidos se perciban mejor que otros en condiciones de dificultad no es solo teórico, puesto que tiene una enorme gama de aplicaciones, donde una de las más próximas al lenguaje es la enseñanza de la lengua (Tessa y Atagi, 2015; Liu y Jin, 2015). Por otra parte, la complejidad del fenómeno lo convierte en punto de encuentro obligado de diversas disciplinas con intereses propios, como la acústica, la psicolingüística y la neurofisiología, toda vez que desafía la memoria y la concentración de los auditores además de constituir un reto que pone a prueba la robustez de las propiedades físicas de los sonidos involucrados.

\section{REFERENCIAS}

Cunningham, J., Nicol, T., Zecker, S. G., Bradlow, A. y Kraus, N. 2001. Neurobiologic responses to speech in noise in children with learning problems: deficits and strategies for improvement. Clinical Neurophysiology, 112(5), 758767.

Léger, A. C., Reed, Ch. M., Desloge, J.G., Swaminathan, J. y Braida, L. D. 2015. Consonant identification in noise using Hilbert-transform temporal finestructure speech and recovered-envelope speech for listeners with normal and impaired hearing. The Journal of the Acoustical Society of America, 138, 389403. http://doi.org/10.1121/1.4922949

Liu, Ch. y Jin, S-H. 2015. Auditory detection of non-speech and speech stimuli in noise: Effects of listeners' native language background. The Journal of the Acoustical Society of America, 138, 2782-2790.

Marrero, V., Rodríguez, M. J. e Igualada, A. 2015. Los efectos del ruido sobre la percepción del habla. En M. A. Penas (ed.). Panorama de fonética española actual. Madrid: Arco/Libros (pp. 367-400). 
Marxer, R., Barker, J., Martin C. y García M.L. 2016. A corpus of noise-induced word misperceptions for English. The Journal of the Acoustical Society of America, 140, 458-463. http://doi.org/10.1121/1.4967185

Nilsson, M., Soli, S., y Sullivan, J. 1994. Development of the Hearing in Noise Test for the measurement of speech reception thresholds in quiet and in noise. The Journal of the Acoustical Society of America, 95(2),1085-1099.

Parikh, G. y Loizou, P. C. 2005. The influency of noise on vowel and consonant cues. The Journal of the Acoustical Society of America, 118, 3874-3888.

Peutz, V.m.A. 1971. Articulation loss of consonants as a criterion for speech transmission in a room. Journal of Audio Engineering Society, 19(11), 23-27.

Rosas, C. y Sommerhoff, J. 2008. Inteligibilidad acústica en español: una propuesta para su medición. Estudios filológicos, 43, 179-190.

Sommerhoff, J. y Rosas, C. 2007. Evaluación de la inteligibilidad del habla en español. Estudios filológicos, 42, 215-225.

Sommerhoff, J. y Rosas, C. 2012. Logatom corpus for the assessment of the intelligibility in Spanish speaking environments and its relation with STI measurements. Applied Acoustics, 73, 1190-1200.

Tessa, B. y Atagi, E. 2015. Children's perception of nonnative-accented sentences in noise and quiet. The Journal of the Acoustical Society of America, 138, 39853993.

Tóth, M. A., García, M. L., Tang, Y. y Cooke, M. 2015. "A corpus of noiseinduced word misperceptions for Spanish". The Journal of the Acoustical Society of America, 137, 2, 184-189. https://doi.org/10.1121/1.4905877 Google Scholar Scitation

Ziegler, J. C., Pech-George, C. y Lorenzi, C. 2009. "Speech perception noise deficits in dyslexia”, Developmental Science, 12, 732-745. 
ANEXO 1.

LISTA DE LOGATOMOS

Lista $\mathrm{N}^{\circ} 1$

\begin{tabular}{|c|c|c|c|c|c|c|}
\hline tun & yib & faf & mum & chak & ram & kad \\
\hline reg & for & fen & mag & bab & dok & non \\
\hline buj & gun & kig & fat & dip & lal & \\
\hline mik & let & jug & pog & kop & bel & \\
\hline gil & yuf & sij & num & dud & lef & \\
\hline mep & yej & yut & sat & geb & jid & \\
\hline sen & ger & kuk & got & jom & nab & \\
\hline taj & tot & pim & bit & gof & loj & \\
\hline nuj & rok & sul & jap & chim & sor & \\
\hline yol & nar & saf & yan & ped & nif & \\
\hline sob & jek & ched & ter & mod & til & \\
\hline kem & teb & bif & chog & deg & tof & \\
\hline pak & ful & fij & dam & lur & gaj & \\
\hline rip & lin & fob & lub & rud & bon & \\
\hline pup & nit & yir & chup & nel & bar & \\
\hline
\end{tabular}

Lista $\mathrm{N}^{\circ} 2$

\begin{tabular}{|c|c|c|c|c|c|c|}
\hline chon & daf & muf & gid & tap & gom & lop \\
\hline dul & jot & rul & lad & sam & dij & jeb \\
\hline tom & seg & ror & ren & pit & rob & \\
\hline luk & nim & yag & dob & yik & yep & \\
\hline ned & mib & lem & jil & raf & pab & \\
\hline lig & koj & bed & fok & mir & gug & \\
\hline fam & mol & kal & nak & puj & kin & \\
\hline bup & mut & yum & tug & nog & jaj & \\
\hline jof & chel & chit & nup & nor & bak & \\
\hline kef & char & bim & mej & pif & gap & \\
\hline bog & dor & feg & chab & jer & chif & \\
\hline yod & gek & tid & nut & fip & chuj & \\
\hline fud & rij & rat & pon & par & man & \\
\hline jun & nef & sud & kur & ket & tek & \\
\hline den & sok & kub & pel & dat & sip & \\
\hline
\end{tabular}




\section{Lista $\mathrm{N}^{\circ} 3$}

\begin{tabular}{|c|c|c|c|c|c|c|}
\hline sug & tuk & ril & dun & beg & tad & gig \\
\hline tig & nud & deb & chen & pob & bip & med \\
\hline fid & chat & jin & chor & bud & jef & \\
\hline pen & kut & yit & neg & fug & dil & \\
\hline kol & jal & chaf & yif & dof & joj & \\
\hline pul & nam & chul & pij & bam & lik & \\
\hline yab & jet & gop & chij & kuf & yuj & \\
\hline ñet & kej & gad & sap & lep & mak & \\
\hline raj & sid & por & mim & kan & reb & \\
\hline mog & sek & nip & nur & fap & yar & \\
\hline nok & tem & jub & kir & lum & rer & \\
\hline pat & yel & som & der & bok & rot & \\
\hline gem & chob & rof & lod & paf & kib & \\
\hline guk & lag & ñf & jur & top & dot & \\
\hline mup & fom & fek & run & yon & daj & \\
\hline
\end{tabular}

\section{Lista $\mathrm{N}^{\circ} 4$}

\begin{tabular}{|c|c|c|c|c|c|c|}
\hline faj & fot & sil & sof & pok & nob & jem \\
\hline gef & bob & lib & baf & tin & gub & nul \\
\hline tal & saj & nij & mon & sun & rid & \\
\hline cham & yak & rom & cheg & kag & kum & \\
\hline lut & bul & kod & rap & rug & sot & \\
\hline chok & lej & juk & fer & lan & kep & \\
\hline mel & lir & chip & nat & mif & mar & \\
\hline jig & fof & ben & jad & yed & bor & \\
\hline dek & fil & kik & yog & nim & nor & \\
\hline nen & gal & fun & tef & mab & did & \\
\hline bij & mit & dug & yim & peg & naf & \\
\hline jop & rek & get & luf & muj & feb & \\
\hline gur & yup & tur & dap & pip & lol & \\
\hline pud & tet & tub & pam & chud & toj & \\
\hline goj & dom & seb & bat & gin & ser & \\
\hline
\end{tabular}




\section{Lista $\mathrm{N}^{\circ} 5$}

\begin{tabular}{|c|c|c|c|c|c|c|}
\hline kit & kon & tol & fur & sub & ber & yok \\
\hline jag & rad & guf & fet & dig & kar & chug \\
\hline sin & gib & mor & rig & jik & mij & \\
\hline nun & pid & chid & baj & gej & gan & \\
\hline sal & men & foj & soj & dop & mul & \\
\hline bof & rem & fin & fal & mob & pom & \\
\hline yud & neb & yeg & kel & fub & bil & \\
\hline tej & nil & sur & chap & naj & chom & \\
\hline chek & nam & rop & not & ner & tib & \\
\hline ruk & kif & maf & gut & pap & bot & \\
\hline set & log & kuj & pug & dad & tir & \\
\hline yip & bun & tuf & mat & beb & tut & \\
\hline yam & fef & lup & tan & dem & nof & \\
\hline lim & pek & jum & kab & sef & jod & \\
\hline led & jep & gir & duk & lak & gol & \\
\hline
\end{tabular}

\section{Lista $\mathrm{N}^{\circ} 6$}

\begin{tabular}{|c|c|c|c|c|c|c|}
\hline fig & lab & dal & mok & cheb & luj & yaf \\
\hline peb & kim & fop & mam & ret & nar & rub \\
\hline chun & bid & pot & bug & pof & kup & \\
\hline gep & mip & tod & tep & nid & sem & \\
\hline chil & juf & meg & chaj & suk & mud & \\
\hline tum & fem & yen & tag & yor & nek & \\
\hline lit & bom & yob & kak & sop & fuk & \\
\hline doj & god & cher & ral & gik & jan & \\
\hline ref & gum & bap & lar & jut & roj & \\
\hline bek & fad & yat & jib & pun & dub & \\
\hline chot & ked & kog & nap & jir & nit & \\
\hline yul & lif & def & jej & rur & dur & \\
\hline yij & nuf & lon & din & tik & det & \\
\hline lel & jol & paj & per & sig & gag & \\
\hline rin & pil & nom & sad & chof & nug & \\
\hline
\end{tabular}




\section{Lista $\mathrm{N}^{\circ} 7$}

\begin{tabular}{|c|c|c|c|c|c|c|}
\hline nuk & lat & dol & jak & gif & len & mid \\
\hline lor & lul & mom & jim & gab & bad & fod \\
\hline dib & rut & dut & sod & duf & jed & \\
\hline tel & jup & bem & chef & kud & kok & \\
\hline pur & ruf & jog & tif & gel & nem & \\
\hline tuj & nop & gon & bop & gar & ran & \\
\hline chub & sik & yot & lij & big & choj & \\
\hline yer & pef & ton & fum & pal & sep & \\
\hline fag & pin & sum & yun & pet & fik & \\
\hline sag & nig & git & rib & dan & tit & \\
\hline tar & lob & laf & kip & rir & chur & \\
\hline mek & yeb & nom & rej & guj & nad & \\
\hline mug & chal & yil & pub & tab & poj & \\
\hline yaj & buk & dej & map & yof & chet & \\
\hline fep & rol & kam & dir & chin & keg & \\
\hline
\end{tabular}

\section{Lista $\mathrm{N}^{\circ} 8$}

\begin{tabular}{|c|c|c|c|c|c|c|}
\hline kob & fan & tak & nal & tup & sol & nef \\
\hline pop & puk & juj & mil & yug & pem & naf \\
\hline sut & fib & mot & mun & boj & mof & \\
\hline bet & mer & kul & fuf & yid & noj & \\
\hline lok & dag & rik & sib & ted & kaf & \\
\hline bur & kij & maj & bin & nub & dep & \\
\hline gim & fol & yap & rum & yom & suf & \\
\hline chop & kor & gup & pig & rod & bef & \\
\hline nir & leg & chad & lam & lud & not & \\
\hline fej & jit & bub & gak & nur & chuk & \\
\hline chig & net & meb & dod & tim & san & \\
\hline jif & fir & pad & nin & rep & jon & \\
\hline jab & sej & chem & dik & yek & jar & \\
\hline ged & rag & gog & ken & bal & kat & \\
\hline lip & dum & tog & jel & fut & sir & \\
\hline
\end{tabular}


Lista $\mathrm{N}^{\circ} 9$

\begin{tabular}{|c|c|c|c|c|c|c|}
\hline gat & dak & tul & sif & muk & pir & yet \\
\hline put & kap & rim & bag & leb & red & ten \\
\hline mig & chir & pol & rup & nep & pej & \\
\hline bum & jeg & yur & nem & pib & laj & \\
\hline mem & jip & bep & mop & rog & jud & \\
\hline bik & kug & fel & yal & kid & ded & \\
\hline sab & gor & puf & chan & ler & suj & \\
\hline yin & lot & yub & lun & chol & fon & \\
\hline chej & fif & chuf & kek & taf & tat & \\
\hline tor & sel & yoj & jam & dog & chib & \\
\hline dup & rak & sit & gij & nod & sar & \\
\hline gob & chut & lil & tij & num & gul & \\
\hline fit & tob & pan & nik & bod & gen & \\
\hline yef & gaf & kom & far & fuj & mad & \\
\hline fab & jok & dim & nag & son & lof & \\
\hline
\end{tabular}

Lista $\mathrm{N}^{\circ} 10$

\begin{tabular}{|c|c|c|c|c|c|c|}
\hline nib & rab & kun & kaj & sak & fak & kot \\
\hline chep & kil & nol & rif & gud & chum & bir \\
\hline tud & nat & ban & dit & ker & jat & \\
\hline geg & nej & duj & sup & jij & lid & \\
\hline dar & rel & keb & del & jen & nuf & \\
\hline yem & nir & jul & chag & dab & pep & \\
\hline but & pag & yop & gok & pod & fed & \\
\hline ruj & met & teg & yuk & lom & sog & \\
\hline tok & lap & sed & ner & nof & chod & \\
\hline mef & rar & jor & lek & bej & tam & \\
\hline mub & fog & tip & pik & gip & fim & \\
\hline yad & dif & yig & nan & don & min & \\
\hline gam & pum & bib & mal & jaf & mur & \\
\hline ron & buf & lug & bol & sim & nut & \\
\hline chik & moj & kof & job & rit & fup & \\
\hline
\end{tabular}

Total $=920$ palabras 


\section{ANEXO 2. \\ PORCENTAJES DE OMISIONES, ERRORES Y ACIERTOS DE LOS LOGATOMOS DICTADOS}

$\begin{array}{rrrr} & & \text { Casos x } & \% \mathrm{x} \\ \text { Casos } & \% \text { Total } & \text { Grupo } & \text { Grupo } \\ 31273 & 100 \% & & \end{array}$

Cons. dictado/

Inicial confundido

b

$\mathrm{b} / \mathrm{d}$

$\mathrm{b} / \mathrm{g}$

$\mathrm{b} / \mathrm{p}$

$\mathrm{b} / \mathrm{k}$

$\mathrm{b} / \mathrm{m}$

b/f

$\mathrm{b} / \mathrm{t}$

b/ll

ch

$\mathrm{ch} / \mathrm{k}$

ch/ll

$\mathrm{ch} / \mathrm{j}$

$\mathrm{ch} / \mathrm{t}$

$\mathrm{ch} / \mathrm{g}$

d

$d / t$

$d / b$

$\mathrm{d} / \mathrm{g}$

$\mathrm{d} / \mathrm{l}$

$\mathrm{d} / \mathrm{k}$

$\mathrm{d} / \mathrm{p}$

$\mathrm{d} / \mathrm{r}$

d/ll

f

f/p

f/j

$\mathrm{f} / \mathrm{s}$

$\mathrm{f} / \mathrm{t}$

f/g

$\mathrm{f} / \mathrm{k}$
Lugar

bilabial

dental

velar

bilabial

velar

bilabial

labiodental fricativa

dental

oclusiva

palatal

palatal

velar

fricativa

africada

oclusiva

palatal

fricativa

velar

dental

fricativa

oclusiva

velar

dental

dental

oclusiva

oclusiva

oclusiva

bilabial

oclusiva

velar

oclusiva

alveolar

lateral

velar

oclusiva

bilabial

oclusiva

alveolar

vibrante

palatal

fricativa

labiodental fricativa

bilabial

oclusiva

velar

alveolar

fricativa

fricativa

dental

oclusiva

velar

oclusiva

velar

oclusiva

Cuerdas

Vocales

723

2,312\%

sonora

sonora

39

0,125\%

55

$0,176 \%$

sonora

0,038\%

$87 \quad 63,218 \%$

sorda

sorda

sonora

sorda

sorda

sonora

sorda

sorda

sonora

sorda

sorda

sonora

sonora

sorda

sonora

0,035\%

0,013\%

0,006\%

0,003\%

0,003\%

0,003\%

13,793\%

$12,644 \%$

$4,598 \%$

$2,299 \%$

$1,149 \%$

$1,149 \%$

$1,149 \%$

24

0,077\%

11

$0,035 \%$

$25 \quad 44,000 \%$

$28,000 \%$

$16,000 \%$

$8,000 \%$

$4,000 \%$

1

$0,003 \%$

41

$0,131 \%$

69

$0,221 \%$

$162 \quad 42,593 \%$

$38,889 \%$

$7,407 \%$

$3,086 \%$

$2,469 \%$

$1,852 \%$

$1,852 \%$

$1,852 \%$

3

$0,010 \%$

sonora

$0,010 \%$

sorda

$0,112 \%$

sorda

$0,122 \%$

$12430,645 \%$

sorda

$0,118 \%$

$29,839 \%$

sorda

sorda

0,058\%

$14,516 \%$

$6,452 \%$

sonora

0,026\%

$5,645 \%$

sorda

$0,022 \%$

$5,645 \%$ 
RLA. Revista de Lingüística Teórica y Aplicada, 55 (2), II Sem. 2017

\begin{tabular}{|c|c|c|c|c|c|c|c|}
\hline $\mathrm{f} / \mathrm{b}$ & bilabial & oclusiva & sonora & 4 & $0,013 \%$ & & $3,226 \%$ \\
\hline $\mathrm{f} / \mathrm{d}$ & dental & oclusiva & sonora & 2 & $0,006 \%$ & & $1,613 \%$ \\
\hline $\mathrm{f} / \mathrm{r}$ & alveolar & vibrante Múlt. & sonora & 2 & $0,006 \%$ & & $1,613 \%$ \\
\hline $\mathrm{f} / \mathrm{ch}$ & palatal & africada & sorda & 1 & $0,003 \%$ & & $0,806 \%$ \\
\hline g & velar & oclusiva & sonora & 54 & $0,173 \%$ & & \\
\hline$g / b$ & bilabial & oclusiva & sonora & 74 & $0,237 \%$ & 205 & $36,098 \%$ \\
\hline g/ll & palatal & fricativa & sonora & 60 & $0,192 \%$ & & $29,268 \%$ \\
\hline $\mathrm{g} / \mathrm{d}$ & dental & oclusiva & sonora & 38 & $0,122 \%$ & & $18,537 \%$ \\
\hline$g / j$ & velar & fricativa & sorda & 14 & $0,045 \%$ & & $6,829 \%$ \\
\hline $\mathrm{g} / \mathrm{r}$ & alveolar & vibrante Múlt. & sonora & 9 & $0,029 \%$ & & $4,390 \%$ \\
\hline $\mathrm{g} / \mathrm{k}$ & velar & oclusiva & sorda & 8 & $0,026 \%$ & & $3,902 \%$ \\
\hline $\mathrm{g} / \mathrm{n}$ & alveolar & nasal & sonora & 1 & $0,003 \%$ & & $0,488 \%$ \\
\hline $\mathrm{g} / \mathrm{s}$ & alveolar & fricativa & sorda & 1 & $0,003 \%$ & & $0,488 \%$ \\
\hline j & velar & fricativa & sorda & 75 & $0,240 \%$ & & \\
\hline $\mathrm{j} / \mathrm{g}$ & velar & oclusiva & sonora & 194 & $0,620 \%$ & 337 & $57,567 \%$ \\
\hline $\mathrm{j} / \mathrm{k}$ & velar & oclusiva & sorda & 64 & $0,205 \%$ & & $18,991 \%$ \\
\hline$j / p$ & bilabial & oclusiva & sorda & 36 & $0,115 \%$ & & $10,682 \%$ \\
\hline$j / f$ & labiodental & fricativa & sorda & 15 & $0,048 \%$ & & $4,451 \%$ \\
\hline$j / r$ & alveolar & vibrante Múlt. & sonora & 9 & $0,029 \%$ & & $2,671 \%$ \\
\hline$j / t$ & dental & oclusiva & sorda & 8 & $0,026 \%$ & & $2,374 \%$ \\
\hline $\mathrm{j} / \mathrm{b}$ & bilabial & oclusiva & sonora & 4 & $0,013 \%$ & & $1,187 \%$ \\
\hline $\mathrm{j} / \mathrm{s}$ & alveolar & fricativa & sorda & 4 & $0,013 \%$ & & $1,187 \%$ \\
\hline$j / 1$ & alveolar & lateral & sonora & 2 & $0,006 \%$ & & $0,593 \%$ \\
\hline $\mathrm{j} / \mathrm{ch}$ & palatal & africada & sorda & 1 & $0,003 \%$ & & $0,297 \%$ \\
\hline $\mathbf{k}$ & velar & oclusiva & sorda & 103 & $0,329 \%$ & & \\
\hline $\mathrm{k} / \mathrm{j}$ & velar & fricativa & sorda & 117 & $0,374 \%$ & 303 & $38,614 \%$ \\
\hline $\mathrm{k} / \mathrm{p}$ & bilabial & oclusiva & sorda & 60 & $0,192 \%$ & & $19,802 \%$ \\
\hline $\mathrm{k} / \mathrm{t}$ & dental & oclusiva & sorda & 56 & $0,179 \%$ & & $18,482 \%$ \\
\hline $\mathrm{k} / \mathrm{g}$ & velar & oclusiva & sonora & 52 & $0,166 \%$ & & $17,162 \%$ \\
\hline $\mathrm{k} / \mathrm{f}$ & labiodental & fricativa & sorda & 7 & $0,022 \%$ & & $2,310 \%$ \\
\hline $\mathrm{k} / \mathrm{ll}$ & palatal & fricativa & sonora & 5 & $0,016 \%$ & & $1,650 \%$ \\
\hline $\mathrm{k} / \mathrm{r}$ & alveolar & vibrante Múlt. & sonora & 4 & $0,013 \%$ & & $1,320 \%$ \\
\hline $\mathrm{k} / \mathrm{b}$ & bilabial & oclusiva & sonora & 1 & $0,003 \%$ & & $0,330 \%$ \\
\hline $\mathrm{k} / \mathrm{d}$ & dental & oclusiva & sonora & 1 & $0,003 \%$ & & $0,330 \%$ \\
\hline 1 & alveolar & lateral & sonora & 22 & $0,070 \%$ & & \\
\hline $1 / \mathrm{n}$ & alveolar & nasal & sonora & 8 & $0,026 \%$ & 22 & $36,364 \%$ \\
\hline $1 / b$ & bilabial & oclusiva & sonora & 3 & $0,010 \%$ & & $13,636 \%$ \\
\hline $1 / d$ & dental & oclusiva & sonora & 3 & $0,010 \%$ & & $13,636 \%$ \\
\hline $1 / \mathrm{g}$ & velar & oclusiva & sonora & 2 & $0,006 \%$ & & $9,091 \%$ \\
\hline
\end{tabular}




\begin{tabular}{|c|c|c|c|c|c|c|c|}
\hline $1 / \mathrm{k}$ & velar & oclusiva & sorda & 2 & $0,006 \%$ & & $9,091 \%$ \\
\hline $1 / \mathrm{m}$ & bilabial & nasal & sonora & 1 & $0,003 \%$ & & $4,545 \%$ \\
\hline $1 / \mathrm{r}$ & alveolar & vibrante Múlt. & sonora & 1 & $0,003 \%$ & & $4,545 \%$ \\
\hline $1 / \mathrm{t}$ & dental & oclusiva & sorda & 1 & $0,003 \%$ & & $4,545 \%$ \\
\hline $1 / 11$ & palatal & fricativa & sonora & 1 & $0,003 \%$ & & $4,545 \%$ \\
\hline $\mathbf{m}$ & bilabial & nasal & sonora & 21 & $0,067 \%$ & & \\
\hline $\mathrm{m} / \mathrm{n}$ & alveolar & nasal & sonora & 44 & $0,141 \%$ & 46 & $95,652 \%$ \\
\hline $\mathrm{m} / \mathrm{b}$ & bilabial & oclusiva & sonora & 1 & $0,003 \%$ & & \\
\hline $\mathrm{m} / \tilde{\mathrm{n}}$ & palatal & nasal & sonora & 1 & $0,003 \%$ & & \\
\hline $\mathbf{n}$ & alveolar & nasal & sonora & 26 & $0,083 \%$ & & \\
\hline $\mathrm{n} / \mathrm{m}$ & bilabial & nasal & sonora & 113 & $0,361 \%$ & 133 & $84,962 \%$ \\
\hline $\mathrm{n} / \tilde{\mathrm{n}}$ & palatal & nasal & sonora & 9 & $0,029 \%$ & & $6,767 \%$ \\
\hline $\mathrm{n} / \mathrm{l}$ & alveolar & lateral & sonora & 8 & $0,026 \%$ & & $6,015 \%$ \\
\hline $\mathrm{n} / \mathrm{d}$ & dental & oclusiva & sonora & 1 & $0,003 \%$ & & $0,752 \%$ \\
\hline$n / j$ & velar & fricativa & sorda & 1 & $0,003 \%$ & & $0,752 \%$ \\
\hline $\mathrm{n} / \mathrm{t}$ & dental & oclusiva & sorda & 1 & $0,003 \%$ & & $0,752 \%$ \\
\hline$\tilde{\mathbf{n}}$ & palatal & nasal & sonora & 10 & $0,032 \%$ & & \\
\hline$\tilde{\mathrm{n}} / \mathrm{m}$ & bilabial & nasal & sonora & 7 & $0,022 \%$ & 16 & $43,750 \%$ \\
\hline$\tilde{n} / \mathrm{n}$ & alveolar & nasal & sonora & 7 & $0,022 \%$ & & $43,750 \%$ \\
\hline$\tilde{\mathrm{n}} / 1$ & alveolar & lateral & sonora & 1 & $0,003 \%$ & & $6,250 \%$ \\
\hline$\tilde{\mathrm{n}} / 11$ & palatal & fricativa & sonora & 1 & $0,003 \%$ & & $6,250 \%$ \\
\hline p & bilabial & oclusiva & sorda & 116 & $0,371 \%$ & & \\
\hline $\mathrm{p} / \mathrm{t}$ & dental & oclusiva & sorda & 65 & $0,208 \%$ & 224 & $29,018 \%$ \\
\hline $\mathrm{p} / \mathrm{j}$ & velar & fricativa & sorda & 48 & $0,153 \%$ & & $21,429 \%$ \\
\hline $\mathrm{p} / \mathrm{f}$ & labiodental & fricativa & sorda & 37 & $0,118 \%$ & & $16,518 \%$ \\
\hline $\mathrm{p} / \mathrm{k}$ & velar & oclusiva & sorda & 24 & $0,077 \%$ & & $10,714 \%$ \\
\hline $\mathrm{p} / \mathrm{b}$ & bilabial & oclusiva & sonora & 20 & $0,064 \%$ & & $8,929 \%$ \\
\hline $\mathrm{p} / \mathrm{d}$ & dental & oclusiva & sonora & 10 & $0,032 \%$ & & $4,464 \%$ \\
\hline $\mathrm{p} / \mathrm{g}$ & velar & oclusiva & sonora & 7 & $0,022 \%$ & & $3,125 \%$ \\
\hline $\mathrm{p} / 1$ & alveolar & lateral & sonora & 4 & $0,013 \%$ & & $1,786 \%$ \\
\hline $\mathrm{p} / \mathrm{s}$ & alveolar & fricativa & sorda & 4 & $0,013 \%$ & & $1,786 \%$ \\
\hline $\mathrm{p} / \mathrm{r}$ & alveolar & vibrante Múlt. & sonora & 3 & $0,010 \%$ & & $1,339 \%$ \\
\hline $\mathrm{p} / \mathrm{m}$ & bilabial & nasal & sonora & 1 & $0,003 \%$ & & $0,446 \%$ \\
\hline $\mathrm{p} / \mathrm{n}$ & alveolar & nasal & sonora & 1 & $0,003 \%$ & & $0,446 \%$ \\
\hline $\mathbf{r}$ & alveolar & $\begin{array}{l}\text { vibrante } \\
\text { Múlt. }\end{array}$ & sonora & 34 & $0,109 \%$ & & \\
\hline $\mathrm{r} / \mathrm{b}$ & bilabial & oclusiva & sonora & 9 & $0,029 \%$ & 16 & $56,250 \%$ \\
\hline $\mathrm{r} / \mathrm{j}$ & velar & fricativa & sorda & 2 & $0,006 \%$ & & $12,500 \%$ \\
\hline$r / t$ & dental & oclusiva & sorda & 2 & $0,006 \%$ & & $12,500 \%$ \\
\hline
\end{tabular}


RLA. Revista de Lingüística Teórica y Aplicada, 55 (2), II Sem. 2017

\begin{tabular}{|c|c|c|c|c|c|c|c|}
\hline $\mathrm{r} / \mathrm{d}$ & dental & oclusiva & sonora & 1 & $0,003 \%$ & & $6,250 \%$ \\
\hline $\mathrm{r} / \mathrm{l}$ & alveolar & lateral & sonora & 1 & $0,003 \%$ & & $6,250 \%$ \\
\hline $\mathrm{r} / \mathrm{p}$ & bilabial & oclusiva & sorda & 1 & $0,003 \%$ & & $6,250 \%$ \\
\hline$s$ & alveolar & fricativa & sorda & 18 & $0,058 \%$ & & \\
\hline$s / j$ & velar & fricativa & sorda & 7 & $0,022 \%$ & 20 & $35,000 \%$ \\
\hline $\mathrm{s} / \mathrm{k}$ & velar & oclusiva & sorda & 5 & $0,016 \%$ & & $25,000 \%$ \\
\hline$s / f$ & labiodental & fricativa & sorda & 3 & $0,010 \%$ & & $15,000 \%$ \\
\hline$s / t$ & dental & oclusiva & sorda & 3 & $0,010 \%$ & & $15,000 \%$ \\
\hline s/g & velar & oclusiva & sonora & 1 & $0,003 \%$ & & $5,000 \%$ \\
\hline$s / r$ & alveolar & vibrante Múlt. & sonora & 1 & $0,003 \%$ & & $5,000 \%$ \\
\hline $\mathbf{t}$ & dental & oclusiva & sorda & 80 & $0,256 \%$ & & \\
\hline $\mathrm{t} / \mathrm{p}$ & bilabial & oclusiva & sorda & 150 & $0,480 \%$ & 271 & $55,351 \%$ \\
\hline $\mathrm{t} / \mathrm{k}$ & velar & oclusiva & sorda & 45 & $0,144 \%$ & & $16,605 \%$ \\
\hline$t / d$ & dental & oclusiva & sonora & 22 & $0,070 \%$ & & $8,118 \%$ \\
\hline$t / j$ & velar & fricativa & sorda & 13 & $0,042 \%$ & & $4,797 \%$ \\
\hline$t / f$ & labiodental & fricativa & sorda & 11 & $0,035 \%$ & & $4,059 \%$ \\
\hline$t / r$ & alveolar & vibrante Múlt. & sonora & 9 & $0,029 \%$ & & $3,321 \%$ \\
\hline$t / s$ & alveolar & fricativa & sorda & 7 & $0,022 \%$ & & $2,583 \%$ \\
\hline $\mathrm{t} / \mathrm{g}$ & velar & oclusiva & sonora & 6 & $0,019 \%$ & & $2,214 \%$ \\
\hline$t / 1$ & alveolar & lateral & sonora & 3 & $0,010 \%$ & & $1,107 \%$ \\
\hline $\mathrm{t} / \mathrm{b}$ & bilabial & oclusiva & sonora & 2 & $0,006 \%$ & & $0,738 \%$ \\
\hline $\mathrm{t} / \mathrm{ch}$ & palatal & africada & sorda & 1 & $0,003 \%$ & & $0,369 \%$ \\
\hline $\mathrm{t} / \mathrm{n}$ & alveolar & nasal & sonora & 1 & $0,003 \%$ & & $0,369 \%$ \\
\hline$t / l 1$ & palatal & fricativa & sonora & 1 & $0,003 \%$ & & $0,369 \%$ \\
\hline 11 & palatal & fricativa & sonora & 40 & $0,128 \%$ & & \\
\hline $11 / g$ & velar & oclusiva & sonora & 54 & $0,173 \%$ & 87 & $62,069 \%$ \\
\hline $11 / j$ & velar & fricativa & sorda & 15 & $0,048 \%$ & & $17,241 \%$ \\
\hline 11/ch & palatal & africada & sorda & 5 & $0,016 \%$ & & $5,747 \%$ \\
\hline $11 / 1$ & alveolar & lateral & sonora & 5 & $0,016 \%$ & & $5,747 \%$ \\
\hline $11 / b$ & bilabial & oclusiva & sonora & 3 & $0,010 \%$ & & $3,448 \%$ \\
\hline $11 / \mathrm{t}$ & dental & oclusiva & sorda & 3 & $0,010 \%$ & & $3,448 \%$ \\
\hline $11 / \mathrm{k}$ & velar & oclusiva & sorda & 1 & $0,003 \%$ & & $1,149 \%$ \\
\hline $11 / \tilde{\mathrm{n}}$ & palatal & nasal & sonora & 1 & $0,003 \%$ & & $1,149 \%$ \\
\hline Total C1 & & & & 2816 & $9,005 \%$ & & \\
\hline $\mathbf{a}$ & central & baja & & & & & \\
\hline$a / o$ & posterior & media & & 9 & $0,029 \%$ & 15 & $60,000 \%$ \\
\hline$a / e$ & anterior & media & & 5 & $0,016 \%$ & & $33,333 \%$ \\
\hline$a / i$ & anterior & alta & & 1 & $0,003 \%$ & & $6,667 \%$ \\
\hline e & anterior & media & & & & & \\
\hline
\end{tabular}




$\begin{array}{lcr}\text { e/a } & \text { central } & \text { baja } \\ \text { e/o } & \text { posterior } & \text { media } \\ \text { e/u } & \text { posterior } & \text { alta } \\ \text { e/i } & \text { anterior } & \text { alta } \\ \mathrm{i} & \text { anterior } & \text { alta } \\ \mathrm{i} / \mathrm{e} & \text { anterior } & \text { media } \\ \mathrm{i} / \mathrm{u} & \text { posterior } & \text { alta } \\ \mathbf{o} & \text { posterior } & \text { media } \\ \mathrm{o} / \mathrm{u} & \text { posterior } & \text { alta } \\ \mathrm{o} / \mathrm{a} & \text { central } & \text { baja } \\ \mathrm{o} / \mathrm{e} & \text { anterior } & \text { media } \\ \mathrm{o} / \mathrm{i} & \text { anterior } & \text { alta } \\ \mathbf{u} & \text { posterior } & \text { alta } \\ \mathrm{u} / \mathrm{i} & \text { anterior } & \text { alta } \\ \mathrm{u} / \mathrm{o} & \text { posterior } & \text { media } \\ \mathrm{u} / \mathrm{a} & \text { central } & \text { baja } \\ \mathrm{u} / \mathrm{e} & \text { anterior } & \text { media } \\ \mathrm{T} & & \end{array}$

Cons.

\begin{tabular}{|c|c|c|c|c|c|c|c|c|}
\hline \multirow[t]{2}{*}{ Final } & b & bilabial & fricativa & sonora & & & \multirow[b]{2}{*}{611} & \multirow[b]{2}{*}{$37,807 \%$} \\
\hline & $\mathrm{b} / \mathrm{d}$ & dental & fricativa & sonora & 231 & $0,739 \%$ & & \\
\hline & $\mathrm{b} / \mathrm{g}$ & velar & fricativa & sonora & 144 & $0,460 \%$ & & $23,568 \%$ \\
\hline & $\mathrm{b} / \mathrm{p}$ & bilabial & oclusiva & sorda & 94 & $0,301 \%$ & & $15,385 \%$ \\
\hline & $\mathrm{b} / \mathrm{m}$ & bilabial & nasal & sonora & 53 & $0,169 \%$ & & $8,674 \%$ \\
\hline & $\mathrm{b} / \mathrm{r}$ & alveolar & vibr. simple & sonora & 31 & $0,099 \%$ & & $5,074 \%$ \\
\hline & $\mathrm{b} / \mathrm{t}$ & dental & oclusiva & sorda & 23 & $0,074 \%$ & & $3,764 \%$ \\
\hline & $\mathrm{b} / \mathrm{n}$ & alveolar & nasal & sonora & 20 & $0,064 \%$ & & $3,273 \%$ \\
\hline & $\mathrm{b} / \mathrm{l}$ & alveolar & lateral & sonora & 7 & $0,022 \%$ & & $1,146 \%$ \\
\hline & $\mathrm{b} / \mathrm{f}$ & labiodental & fricativa & sorda & 6 & $0,019 \%$ & & $0,982 \%$ \\
\hline & $\mathrm{b} / \mathrm{k}$ & velar & fricativa & sonora & 2 & $0,006 \%$ & & $0,327 \%$ \\
\hline & d & dental & fricativa & sonora & & & & \\
\hline & $d / t$ & dental & oclusiva & sorda & 314 & $1,004 \%$ & 792 & $39,646 \%$ \\
\hline & $d / b$ & bilabial & fricativa & sonora & 177 & $0,566 \%$ & & $22,348 \%$ \\
\hline & $\mathrm{d} / \mathrm{g}$ & velar & fricativa & sonora & 122 & $0,390 \%$ & & $15,404 \%$ \\
\hline & $d / r$ & alveolar & vibr. simple & sonora & 63 & $0,201 \%$ & & $7,955 \%$ \\
\hline & $\mathrm{d} / \mathrm{n}$ & alveolar & nasal & sonora & 38 & $0,122 \%$ & & $4,798 \%$ \\
\hline & $\mathrm{d} / \mathrm{p}$ & bilabial & oclusiva & sorda & 29 & $0,093 \%$ & & $3,662 \%$ \\
\hline & $\mathrm{d} / \mathrm{l}$ & alveolar & lateral & sonora & 21 & $0,067 \%$ & & $2,652 \%$ \\
\hline & $\mathrm{d} / \mathrm{k}$ & velar & fricativa & sonora & 10 & $0,032 \%$ & & $1,263 \%$ \\
\hline
\end{tabular}


RLA. Revista de Lingüística Teórica y Aplicada, 55 (2), II Sem. 2017

\begin{tabular}{|c|c|c|c|c|c|c|c|}
\hline $\mathrm{d} / \mathrm{m}$ & bilabial & nasal & sonora & 9 & $0,029 \%$ & & $1,136 \%$ \\
\hline$d / f$ & labiodental & fricativa & sorda & 8 & $0,026 \%$ & & $1,010 \%$ \\
\hline$d / j$ & velar & fricativa & sorda & 1 & $0,003 \%$ & & $0,126 \%$ \\
\hline f & labiodental & fricativa & sorda & & & & \\
\hline$f / b$ & bilabial & fricativa & sonora & 17 & $0,054 \%$ & 85 & $20,000 \%$ \\
\hline $\mathrm{f} / \mathrm{g}$ & velar & fricativa & sonora & 14 & $0,045 \%$ & & $16,471 \%$ \\
\hline $\mathrm{f} / \mathrm{r}$ & alveolar & vibr. simple & sonora & 12 & $0,038 \%$ & & $14,118 \%$ \\
\hline$f / k$ & velar & fricativa & sonora & 11 & $0,035 \%$ & & $12,941 \%$ \\
\hline$f / d$ & dental & fricativa & sonora & 7 & $0,022 \%$ & & $8,235 \%$ \\
\hline$f / t$ & dental & oclusiva & sorda & 7 & $0,022 \%$ & & $8,235 \%$ \\
\hline$f / j$ & velar & fricativa & sorda & 6 & $0,019 \%$ & & $7,059 \%$ \\
\hline $\mathrm{f} / \mathrm{p}$ & bilabial & oclusiva & sorda & 6 & $0,019 \%$ & & $7,059 \%$ \\
\hline $\mathrm{f} / \mathrm{s}$ & alveolar & fricativa & sorda & 4 & $0,013 \%$ & & $4,706 \%$ \\
\hline$f / l$ & alveolar & lateral & sonora & 1 & $0,003 \%$ & & $1,176 \%$ \\
\hline g & velar & fricativa & sonora & & & & \\
\hline$g / b$ & bilabial & fricativa & sonora & 214 & $0,684 \%$ & 527 & $40,607 \%$ \\
\hline$g / d$ & dental & fricativa & sonora & 136 & $0,435 \%$ & & $25,806 \%$ \\
\hline$g / k$ & velar & fricativa & sonora & 91 & $0,291 \%$ & & $17,268 \%$ \\
\hline$g / r$ & alveolar & vibr. simple & sonora & 28 & $0,090 \%$ & & $5,313 \%$ \\
\hline$g / p$ & bilabial & oclusiva & sorda & 27 & $0,086 \%$ & & $5,123 \%$ \\
\hline$g / t$ & dental & oclusiva & sorda & 13 & $0,042 \%$ & & $2,467 \%$ \\
\hline$g / n$ & alveolar & nasal & sonora & 6 & $0,019 \%$ & & $1,139 \%$ \\
\hline $\mathrm{g} / \mathrm{m}$ & bilabial & nasal & sonora & 5 & $0,016 \%$ & & $0,949 \%$ \\
\hline$g / 1$ & alveolar & lateral & sonora & 4 & $0,013 \%$ & & $0,759 \%$ \\
\hline$g / f$ & labiodental & fricativa & sorda & 2 & $0,006 \%$ & & $0,380 \%$ \\
\hline$g / i$ & anterior & alta & & 1 & $0,003 \%$ & & $0,190 \%$ \\
\hline j & velar & fricativa & sorda & & & & \\
\hline$j / g$ & velar & fricativa & sonora & 354 & $1,132 \%$ & 479 & $73,904 \%$ \\
\hline$j / k$ & velar & fricativa & sonora & 60 & $0,192 \%$ & & $12,526 \%$ \\
\hline$j / f$ & labiodental & fricativa & sorda & 20 & $0,064 \%$ & & $4,175 \%$ \\
\hline$j / b$ & bilabial & fricativa & sonora & 13 & $0,042 \%$ & & $2,714 \%$ \\
\hline$j / t$ & dental & oclusiva & sorda & 11 & $0,035 \%$ & & $2,296 \%$ \\
\hline$j / r$ & alveolar & vibr. simple & sonora & 7 & $0,022 \%$ & & $1,461 \%$ \\
\hline$j / d$ & dental & fricativa & sonora & 5 & $0,016 \%$ & & $1,044 \%$ \\
\hline$j / p$ & bilabial & oclusiva & sorda & 5 & $0,016 \%$ & & $1,044 \%$ \\
\hline$j / s$ & alveolar & fricativa & sorda & 2 & $0,006 \%$ & & $0,418 \%$ \\
\hline $\mathrm{j} / \mathrm{l}$ & alveolar & lateral & sonora & 1 & $0,003 \%$ & & $0,209 \%$ \\
\hline$j / n$ & alveolar & nasal & sonora & 1 & $0,003 \%$ & & $0,209 \%$ \\
\hline $\mathbf{k}$ & velar & fricativa & sonora & & & & \\
\hline
\end{tabular}




\begin{tabular}{|c|c|c|c|c|c|c|c|}
\hline $\mathrm{k} / \mathrm{g}$ & velar & fricativa & sonora & 42 & $0,134 \%$ & 66 & $63,636 \%$ \\
\hline $\mathrm{k} / \mathrm{t}$ & dental & oclusiva & sorda & 12 & $0,038 \%$ & & $18,182 \%$ \\
\hline $\mathrm{k} / \mathrm{p}$ & bilabial & oclusiva & sorda & 5 & $0,016 \%$ & & $7,576 \%$ \\
\hline $\mathrm{k} / \mathrm{b}$ & bilabial & fricativa & sonora & 2 & $0,006 \%$ & & $3,030 \%$ \\
\hline $\mathrm{k} / \mathrm{j}$ & velar & fricativa & sorda & 2 & $0,006 \%$ & & $3,030 \%$ \\
\hline $\mathrm{k} / \mathrm{d}$ & dental & fricativa & sonora & 1 & $0,003 \%$ & & $1,515 \%$ \\
\hline $\mathrm{k} / \mathrm{l}$ & alveolar & lateral & sonora & 1 & $0,003 \%$ & & $1,515 \%$ \\
\hline $\mathrm{k} / \mathrm{r}$ & alveolar & vibr. simple & sonora & 1 & $0,003 \%$ & & $1,515 \%$ \\
\hline 1 & alveolar & lateral & sonora & & & & \\
\hline $1 / \mathrm{k}$ & velar & fricativa & sonora & 6 & $0,019 \%$ & 13 & $46,154 \%$ \\
\hline $1 / \mathrm{n}$ & alveolar & nasal & sonora & 3 & $0,010 \%$ & & $23,077 \%$ \\
\hline $1 / \mathrm{t}$ & dental & oclusiva & sorda & 3 & $0,010 \%$ & & $23,077 \%$ \\
\hline $1 / \mathrm{g}$ & velar & fricativa & sonora & 1 & $0,003 \%$ & & $7,692 \%$ \\
\hline m & bilabial & nasal & sonora & & & & \\
\hline $\mathrm{m} / \mathrm{n}$ & alveolar & nasal & sonora & 267 & $0,854 \%$ & 287 & $93,031 \%$ \\
\hline $\mathrm{m} / \mathrm{b}$ & bilabial & fricativa & sonora & 9 & $0,029 \%$ & & $3,136 \%$ \\
\hline $\mathrm{m} / \mathrm{g}$ & velar & fricativa & sonora & 4 & $0,013 \%$ & & $1,394 \%$ \\
\hline $\mathrm{m} / \mathrm{l}$ & alveolar & lateral & sonora & 2 & $0,006 \%$ & & $0,697 \%$ \\
\hline $\mathrm{m} / \mathrm{p}$ & bilabial & oclusiva & sorda & 2 & $0,006 \%$ & & $0,697 \%$ \\
\hline $\mathrm{m} / \mathrm{r}$ & alveolar & vibr. simple & sonora & 2 & $0,006 \%$ & & $0,697 \%$ \\
\hline $\mathrm{m} / \mathrm{j}$ & velar & fricativa & sorda & 1 & $0,003 \%$ & & $0,348 \%$ \\
\hline n & alveolar & nasal & sonora & & & & \\
\hline $\mathrm{n} / \mathrm{m}$ & bilabial & nasal & sonora & 247 & $0,790 \%$ & 279 & $88,530 \%$ \\
\hline $\mathrm{n} / \mathrm{l}$ & alveolar & lateral & sonora & 28 & $0,090 \%$ & & $10,036 \%$ \\
\hline $\mathrm{n} / \mathrm{r}$ & alveolar & vibr. simple & sonora & 2 & $0,006 \%$ & & $0,717 \%$ \\
\hline $\mathrm{n} / \mathrm{b}$ & bilabial & fricativa & sonora & 1 & $0,003 \%$ & & $0,358 \%$ \\
\hline $\mathrm{n} / \mathrm{d}$ & dental & fricativa & sonora & 1 & $0,003 \%$ & & $0,358 \%$ \\
\hline $\mathbf{p}$ & bilabial & oclusiva & sorda & & & & \\
\hline $\mathrm{p} / \mathrm{b}$ & bilabial & fricativa & sonora & 306 & $0,978 \%$ & 787 & $38,882 \%$ \\
\hline $\mathrm{p} / \mathrm{k}$ & velar & fricativa & sonora & 224 & $0,716 \%$ & & $28,463 \%$ \\
\hline $\mathrm{p} / \mathrm{t}$ & dental & oclusiva & sorda & 89 & $0,285 \%$ & & $11,309 \%$ \\
\hline $\mathrm{p} / \mathrm{g}$ & velar & fricativa & sonora & 70 & $0,224 \%$ & & $8,895 \%$ \\
\hline $\mathrm{p} / \mathrm{f}$ & labiodental & fricativa & sorda & 40 & $0,128 \%$ & & $5,083 \%$ \\
\hline $\mathrm{p} / \mathrm{d}$ & dental & fricativa & sonora & 36 & $0,115 \%$ & & $4,574 \%$ \\
\hline $\mathrm{p} / \mathrm{r}$ & alveolar & vibr. simple & sonora & 15 & $0,048 \%$ & & $1,906 \%$ \\
\hline$p / j$ & velar & fricativa & sorda & 4 & $0,013 \%$ & & $0,508 \%$ \\
\hline $\mathrm{p} / \mathrm{l}$ & alveolar & lateral & sonora & 1 & $0,003 \%$ & & $0,127 \%$ \\
\hline $\mathrm{p} / \mathrm{o}$ & posterior & media & & 1 & $0,003 \%$ & & $0,127 \%$ \\
\hline $\mathrm{p} / \mathrm{s}$ & alveolar & fricativa & sorda & 1 & $0,003 \%$ & & $0,127 \%$ \\
\hline
\end{tabular}


RLA. Revista de Lingüística Teórica y Aplicada, 55 (2), II Sem. 2017

\begin{tabular}{|c|c|c|c|c|c|c|c|}
\hline $\mathbf{r}$ & alveolar & vibr. Múltiple & sonora & & & & \\
\hline $\mathrm{r} / \mathrm{g}$ & velar & fricativa & sonora & 26 & $0,083 \%$ & 73 & $35,616 \%$ \\
\hline $\mathrm{r} / \mathrm{b}$ & bilabial & fricativa & sonora & 22 & $0,070 \%$ & & $30,137 \%$ \\
\hline $\mathrm{r} / \mathrm{d}$ & dental & fricativa & sonora & 13 & $0,042 \%$ & & $17,808 \%$ \\
\hline$r / l$ & alveolar & lateral & sonora & 3 & $0,010 \%$ & & $4,110 \%$ \\
\hline $\mathrm{r} / \mathrm{m}$ & bilabial & nasal & sonora & 3 & $0,010 \%$ & & $4,110 \%$ \\
\hline$r / f$ & labiodental & fricativa & sorda & 2 & $0,006 \%$ & & $2,740 \%$ \\
\hline $\mathrm{r} / \mathrm{m}$ & bilabial & nasal & sonora & 2 & $0,006 \%$ & & $2,740 \%$ \\
\hline$r / j$ & velar & fricativa & sorda & 1 & $0,003 \%$ & & $1,370 \%$ \\
\hline $\mathrm{r} / \mathrm{p}$ & bilabial & oclusiva & sorda & 1 & $0,003 \%$ & & $1,370 \%$ \\
\hline $\mathbf{t}$ & dental & oclusiva & sorda & & & & \\
\hline $\mathrm{t} / \mathrm{d}$ & dental & fricativa & sonora & 132 & $0,422 \%$ & 228 & $57,895 \%$ \\
\hline $\mathrm{t} / \mathrm{k}$ & velar & fricativa & sonora & 40 & $0,128 \%$ & & $17,544 \%$ \\
\hline $\mathrm{t} / \mathrm{g}$ & velar & fricativa & sonora & 19 & $0,061 \%$ & & $8,333 \%$ \\
\hline$t / f$ & labiodental & fricativa & sorda & 15 & $0,048 \%$ & & $6,579 \%$ \\
\hline $\mathrm{t} / \mathrm{p}$ & bilabial & oclusiva & sorda & 10 & $0,032 \%$ & & $4,386 \%$ \\
\hline$t / b$ & bilabial & fricativa & sonora & 7 & $0,022 \%$ & & $3,070 \%$ \\
\hline$t / r$ & alveolar & vibr. simple & sonora & 4 & $0,013 \%$ & & $1,754 \%$ \\
\hline$t / s$ & alveolar & fricativa & sorda & 1 & $0,003 \%$ & & $0,439 \%$ \\
\hline Total C2 & & & & 4227 & $13,516 \%$ & & \\
\hline
\end{tabular}

